\title{
Effects of emotion and semantic relatedness on recognition memory: Behavioral and
}

\section{electrophysiological evidence}

Meng $\mathrm{Han}^{1}$, Bingcan $\mathrm{Li}^{1}, *$ Chunyan $\mathrm{Guo}^{1}, \& *$ Roni Tibon ${ }^{2}$

${ }^{1}$ Beijing Key Laboratory of Learning and Cognition, School of Psychology, Capital Normal University, Beijing, 100048, PR China

${ }^{2}$ MRC Cognition \& Brain Sciences Unit, University of Cambridge, UK, CB2 7EF

*Corresponding authors:

Roni Tibon

MRC Cognition \& Brain Sciences Unit, University of Cambridge

15 Chaucer Road, Cambridge, CB2 7EF, UK

Telephone: +44 1223355 294, Email: roni.tibon@mrc-cbu.cam.ac.uk

Chunyan Guo

School of Psychology, Capital Normal University

No.23 Baiduizijia, Fuwaidajie St. Haidian District, Beijing, 100048 PR China

Email: guocy@cnu.edu.cn

RUNNING TITLE: EMOTIONS AND RELATEDNESS MODULATE MEMORY

Manuscript pages: $52 \quad$ Tables: $2 \quad$ Figures: 3 


\section{Abstract}

Some aspects of our memory are enhanced by emotion, whereas other can be unaffected or even hinder. In particular, previous studies report impaired associative memory of emotional content, an effect termed associative "emotional interference". The current study utilized EEG and an associative recognition paradigm to investigate the cognitive and neural mechanisms associated with this effect. In two experiments, participants studied negative and neutral stimulus-pairs that were either semantically related or unrelated. In Experiment 1 emotions were relevant to the encoding task (valence judgment) whereas in Experiment 2 emotions were irrelevant (familiarity judgment). In a subsequent associative recognition test, EEG was recorded while participants discriminated between intact, rearranged, and new pairs. An associative emotional interference effect was observed in both experiments, but was attenuated for semantically related pairs when emotions were relevant to the study task. Moreover, a modulation of an early associative memory ERP component depended on task relevancy of emotions, and occurred for negative pairs when emotions were relevant, but for semantically related pairs when emotions were irrelevant. A later ERP component depicted a more general pattern, and was observed in all experimental conditions. These results suggest that both emotions and semantic relations can act as organizing principles that promote associative binding. Their ability to contribute to successful retrieval depends on specific task demands.

Keywords: emotion, valence, semantic relatedness, associative recognition, recollection, familiarity, unitization 


\section{Introduction}

Convergent evidence indicate that emotions influence memory, but the ways in which they operate and manifest require further specification (Easterbrook, 1959; Kensinger, 2009; Mackay et al., 2004; Mather \& Sutherland, 2011). The term emotionally enhanced memory (EEM) refers to the common finding that emotional events are remembered better than neutral ones; a phenomenon that had been demonstrated for memory of emotional words, pictures, and stories (e.g., Bradley et al., 1992, with pictorial stimuli; Cahill \& McGaugh, 1995, with stories; and LaBar \& Phelps, 1998, with taboo words). According to one suggestion (Kensinger, 2009), this advantage for emotional content, and negative content in particular, arises from increased engagement of sensory processes, leading to focused attention on intrinsic details and to focal enhancement of the negative event. A similar account is the object-based framework (see also arousal-biased competition; Mather \& Sutherland, 2011), which posits that emotional objects attract attention that enhances within-item binding of their constituent features (Mather, 2007). In support of these suggestions, studies have shown that emotion facilitates memory for within-object memory binding (Kensinger, 2009; Mather \& Nesmith, 2008; Mather \& Sutherland, 2011; Nashiro \& Mather, 2011; Schmidt et al., 2011; Steinmetz et al., 2015).

In contrast to the facilitative effects of emotion on within-item binding, inter-item associative memory (i.e., remembering that two or more items were encoded together) for emotional content is often hinder, an effect termed associative "emotional interference" (Madan et al., 2012; Mao et al., 2015; Mather \& Knight, 2008; Pierce \& Kensinger, 2011; Rimmele et al., 2011). One method of accessing associative episodic memory is recognition, i.e., the judgment that currently presented items were previously experienced together in a specific episodic context. In an associative recognition task, during an initial study phase, participants memorize pairs of stimuli. Then, at a subsequent test phase, 
they are asked to discriminate between intact (studied pairs in the study phase), rearranged (studied items with new combination), or new pairs (of unstudied items). Thus, successful performance require memory for the specific pairings of the stimuli that had been encoded. Pierce and Kensinger (2011) utilized this task to investigate the effects of emotion on associative memory. In their version of the task, studied pairs were comprised of either emotionally arousing or neutral words-pairs. Whereas for intact pairs accuracy did not differ, emotional rearranged word-pairs (and negative ones in particular) were less likely to be correctly identified as rearranged compared to neutral word-pairs. This interaction between emotional effects and retrieval conditions points to the multiplicity of processes involved in associative recognition, and their varying sensitivity to the influence of emotional content.

The prevalent dual-process model of recognition memory suggests that recognition involves two separable processes: familiarity and recollection. Familiarity is a general feeling of encountering something or someone which was previously seen, without memory of additional details from that initial encounter, whereas recollection involves the conscious retrieval of additional contextual details (for review see Yonelinas, 2002). Amongst other evidence, this distinction is supported by a large corpus of event-related potential (ERP) studies, revealing two distinct components which are differentially engaged in recognition judgments. The first component is considered to be the electrophysiological index of familiarity. It onsets $300-500 \mathrm{~ms}$ post-stimuli presentation, elicits greater negativity for new vs. old stimuli, and is prominent over mid-frontal sites. The second component is considered to be an electrophysiological index of recollection. It onsets 500-800 ms post-stimuli presentation, elicits greater positivity to old vs. new stimuli, and is usually (though not always) prominent over parietal sites (reviewed by Curran, 2000; Diana et al., 2007; Rugg \& Curran, 2007).

Previous studies investigating the effects of emotion on these ERP components yielded mixed 
results. Several studies utilized a context effect paradigm, in which neutral stimuli were encoded in the context of a neutral/negative sentences (Maratos \& Rugg, 2001) or scenes (Smith et al., 2004; VenturaBort et al., 2016), and were subsequently discriminated from new stimuli via an old/new recognition judgment task. In one report (Ventura-Bort et al., 2016), the valence of the encoding context modulated the early and late old/new ERP effects. In another (Maratos \& Rugg, 2001), a similar modulation was observed but only in one (out of two) of the experiments reported in the study. Conversely, in a third report (Smith et al., 2004), neither the early old/new component, nor the late one, were modulated by valence, but instead additional early ERP components were elicited for items encoded in emotional contexts. In addition, several studies that employed a source-memory paradigm, in which participants are explicitly asked about the encoding context, reported increased modulation of the old/new ERP effects for emotional contexts (Minor \& Herzmann, 2019; Newsome et al., 2012), whereas others reported an opposite pattern (Mao et al., 2015). Finally, using subjective measures of familiarity and recollection (via remember/know or confidence judgments), several studies reported stronger recollection for emotional vs. neutral stimuli, accompanied by larger ERP old/new effects for the former (Schaefer et al., 2011; Ventura-Bort et al., 2016; Weymar et al., 2010; Weymar et al., 2011). In the current study, we utilized an associative recognition task, together with the well-established distinction between familiarity and recollection, and the putative electrophysiological markers corresponding to these processes, to aid our understanding of the mechanisms that drive emotional effects on associative memory.

Furthermore, the current study sought to examine how pre-existing relations between episodically associated stimuli interact with emotional effects on associative memory. Semantic relatedness effects can manifest in two different ways. First, congruent stimuli combinations, such as related (vs. unrelated) 
pictures, support high Levels of Processing (LOP; Craik \& Lockhart, 1972), yielding rich and elaborated encoding. In turn, during retrieval, this elaborated encoding supports high levels of recollection (summarized by Craik, 2002). Second, stimuli that share pre-existing relations can be subjected to unitization - an encoding process by which several discrete items are perceived and encoded as one single unit (reviewed by Mecklinger \& Jäger, 2009; Yonelinas et al., 2010)—and to be subsequently retrieved via familiarity (e.g., Li et al., 2019; Tibon et al., 2014; Zheng et al., 2015). This latter suggestion is rooted in the idea that instead of creating inter-item links between the items, unitization results in a joint representation of the encoded items (that is, a single item, rather than several linked items). This notion had been demonstrated in several ERP studies, in which the putative electrophysiological correlate of familiarity — the early old/new effect—was observed for episodically formed associations between semantically related stimuli, but not otherwise (Li, et al., 2019; Rhodes \& Donaldson, 2007; Tibon et al., 2014; Tibon, Gronau, et al., 2014; Zheng et al., 2015). The possibility that unitization might support the manifestation of semantic relatedness effects is especially intriguing given the emotional enhancement effect of within-object memory binding (Mather \& Sutherland, 2011). More specifically, it has been argued that when items are unitized, rather than relying on inter-item binding, their relations are supported by links that are qualitatively similar to within-object links (e.g., Mayes et al., 2007; Tibon et al., 2018). Therefore, unitized associations might be subjected to the enhancing effects of emotions on item memory. In other words, if emotions benefit within-object binding, then unitization of emotional stimuli might alleviate the negative effects of emotion on associative memory. Indeed, previous studies showed that when several items were integrated together (e.g., by creating a mental image of the words comprising a pair interacting with each other. For example, for the word pair "flies-booger": imagining flies stuck in booger), associative emotional interference 
can disappear or even transform into mnemonic benefits (e.g., Guillet \& Arndt, 2009; Murray \& Kensinger, 2012).

Finally, the current study examined whether emotional and relatedness effects on associative memory depend on the relevancy of emotions to the encoding task. This point is further elaborated below, but in short, previous studies suggested that emotional content effects on processing and behavior often emerge when emotions are relevant to the task that is performed (e.g., Engen et al., 2017; Huang et al., 2008; Pessoa, 2009; Stein et al., 2009; Wei et al., 2015), though it is unclear whether this also applies to memory. Accordingly, in the present study we conducted two experiments, in which emotions were either relevant (Experiment 1) or irrelevant (Experiment 2) to the encoding task. In both experiments, an associative memory paradigm was used in order to examine the effects of valence (negative, neutral) and relatedness (related, unrelated) of memoranda on associative recognition.

\section{Experiment 1}

In experiment 1, participants memorized and retrieved picture-picture pairs while EEG was recorded. Half the pairs were of negative objects (e.g., gun, spider) and half of neutral objects (e.g., bunny, traffic light). In addition, half the pairs contained objects that were semantically related (e.g., bunny-carrot), whereas half contained objects with no pre-existing semantic relations (e.g., spiderbullet). During study, participants performed a valence judgment task, and at test they were asked to discriminate between intact, rearranged and new pairs.

We predicted an overall reduction in associative memory accuracy for negative vs. neutral stimuli due to associative emotional interference. Importantly, possible moderation of this effect by semantic 
relatedness will crucially depend on the mnemonic processes promoted by semantic relations. More specifically, if semantic relations promote unitization, then associative retrieval of semantically related pairs would benefit from within-item binding, which might attenuate associative emotional interference for negative pairs that are semantically related (compared to unrelated). We therefore predicted that semantic relatedness will attenuate the associative emotional interference for negative-related vs. negative-unrelated pairs, such that associative memory for negative-related pairs would be similar (if not comparable) to memory for neutral-related pairs. This attenuation will be accompanied by a modulation of the early ERP component - the electrophysiological marker of familiarity — for related but not for unrelated pairs, such that the early associative memory ERP effect would be greater for related than for unrelated pairs. If, on the other hand, relatedness increases LOP, then we would not necessarily expect attenuation of the associative emotional interference effect (indeed, we might even observe the opposite pattern). Moreover, we would expect increased modulation of the late ERP, which index recollection, for related compared to unrelated pairs (that is, the late associative memory ERP effect would be greater for related than unrelated pairs).

\section{Method}

Participants

To determine the required sample size, we first extracted the behavioral effect size obtained by Pierce \& Kensinger $(2011 ; \mathrm{N}=32$, Cohen's $\mathrm{f}=0.42)$, who employed a similar experimental design to the one used in the current study. Next, we extracted the associative memory ERP effects (for the early and late ERP components) from multiple studies that used an associative memory paradigm (Bader \& Mecklinger, 2017; Kamp et al., 2016; Li et al., 2017; 2019; Rhodes \& Donaldson, 2009; Tibon, et al., 
2014; Zhao et al., 2020; Zheng et al., 2015). The effect sizes for the associative memory component reported in these studies were all medium-large, ranging from Cohen's $f=0.3$ to 10.7 ( $\mathrm{N}$ ranging between 17 and 46). Nevertheless, to avoid an overinflated estimation of effect size, we set $\mathrm{f}=0.25$ as a lower, more conservative value. Based on this effect size, we estimated that power $>.8(\alpha=.01)$ would require at least 39 participants (actual power $=.81$ ), and therefore recruited 47 participants for the study.

Forty-seven healthy, right-handed native Chinese speakers (30 females; mean age $22.4 \pm 2$ years) from Capital Normal University participated in the experiment and were paid ¥30 per hour. All participants had normal or corrected-to-normal vision, were prescreened for history of neurological or psychiatric disorders, learning disorders, head injury or psychotropic drug use. Informed consent, approved by the Capital Normal University Institutional Review Board, was collected from each participant. Data from five participants were discarded, including one participant with very poor task performance (associative $\operatorname{Pr}<0$ ), and four participants with insufficient number of artifact-free ERP trials in one or more experimental conditions $(\mathrm{N}<16)$. Our final sample therefore included 42 participants (27 females; mean age $22.4 \pm 2$ years).

Stimuli

Our initial stimuli dataset included 1469 object pictures of animals, food, equipment, tools, appliances, etc., from the Hemera Photo-Objects Collection (Hemera Photo Objects, Gatineau, Quebec, Canada), the International Affective Pictures System (Lang et al., 2008) and free internet sources. Colored objects were matched for size, luminance and contrast by using adjustment curve in Adobe Photoshop 8.0, and were presented at the center of the picture on a gray background (RGB: 150; see Fig. 1 for examples). 
bioRxiv preprint doi: https://doi.org/10.1101/2020.02.28.969659; this version posted October 4, 2021. The copyright holder for this preprint (which was not certified by peer review) is the author/funder, who has granted bioRxiv a license to display the preprint in perpetuity. It is made available under aCC-BY 4.0 International license.

A

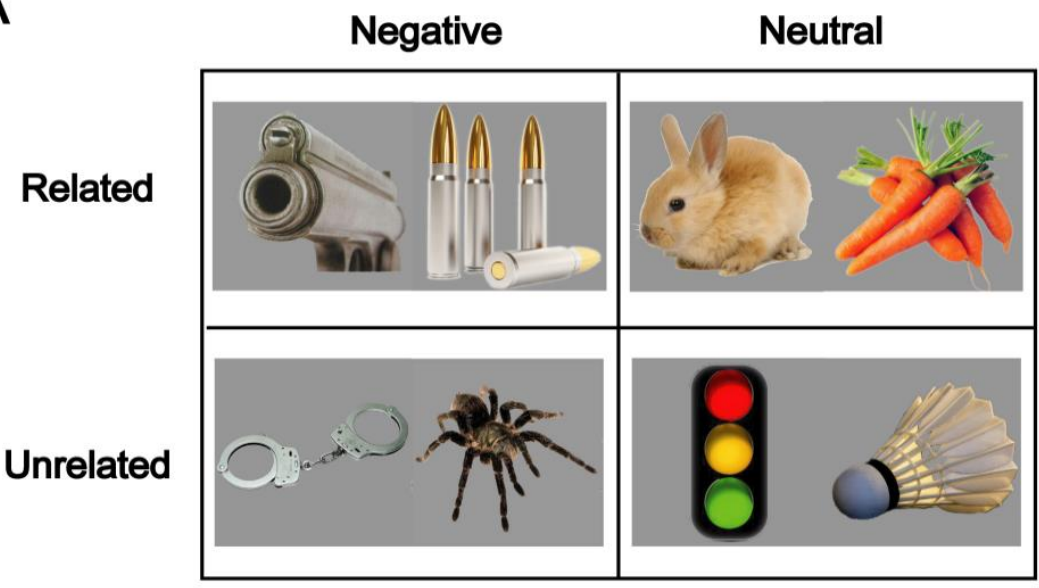

B

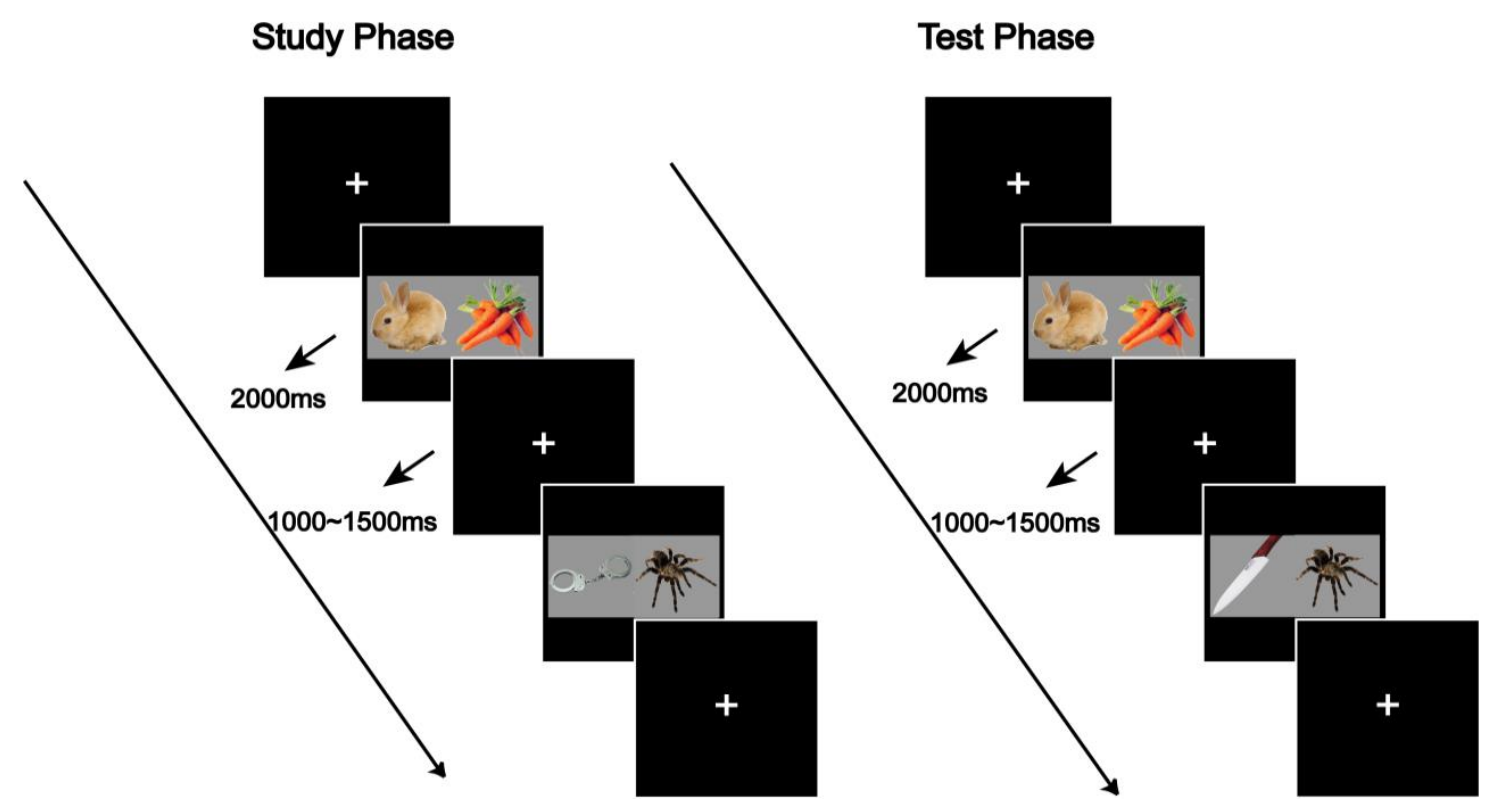

Figure 1. Example stimuli and experimental paradigm. (A) Example stimuli for each condition. Our factorial design included four types of picture pairs, varying in their relatedness (related, unrelated) and valence (negative, neutral). (B) Schematic illustration of the experimental paradigm. At study, participants viewed picture-pairs and indicated which item is more negative (Experiment 1) or more familiar (Experiment 2). At test, they discriminated between intact, rearranged, and new pairs. 
An independent sample $(\mathrm{N}=12)$ provided ratings of the pictures on a scale of 1 (very negative/calm/unfamiliar) to 9 (very positive/exciting/familiar) on the dimensions of valence, arousal and familiarity. Pictures with familiarity ratings below 4 were removed from the pool. 1272 pictures, including 646 negative pictures (with valence score below 4) and 625 neutral pictures (with valence score between 4 and 7), were chosen and combined to form negative/negative picture pairs or neutral/neutral picture pairs, resulting in 166 semantically-unrelated negative, 150 semanticallyunrelated neutral, 157 semantically-related negative, and 162 semantically-related neutral picture pairs. Another independent sample $(\mathrm{N}=10)$ provided ratings of the pairs on the dimension of relatedness. They were asked to judge how likely it is for the two objects to appear together (Tibon et al., 2014), by responding on a scale ranging from 1 (very unlikely) to 9 (very likely). Only pairs for which the preassigned relatedness status was verified (i.e., unrelated stimuli that with relatedness score $<5$, and related stimuli with relatedness score $>=5$ ) by the majority of the raters (at least $6 / 10$ raters) were included in the study. Based on the ratings, 600 pairs were selected, including 150 semantically-related negative pairs, 150 semantically-related neutral pairs, 150 semantically-unrelated negative pairs, and 150 semantically-unrelated neutral pairs. Semantically-related pairs either belonged to the same category (e.g., "desk-sofa"), or were functionally related (e.g., "rabbit-carrot"). The relatedness scores of related pairs $[$ Mean $(\mathrm{SD})=6.61(1.25)]$ were significantly higher than that of unrelated pairs [Mean $(\mathrm{SD})=1.61(.41) ; t(299)=64.20, p<.001]$. Negative pictures were significantly more negative and arousing than neutral pictures [valence: $t(599)=-115.45, p<.001$; arousal: $t(599)=52.36, p<.001$ ], but equal to neutral pictures on familiarity $(p>.05)$.

We subsequently constructed 600 rearranged pairs by combining together pictures belonging to different pairs, but keeping their type and location unchanged, such that there were 150 rearranged pairs 
for each type. For example, two related neutral pairs A-B (e.g., rabbit-carrot) and C-D (e.g., goatcabbage) could be recombined to form another related neutral pair A-D (rabbit-cabbage). B and C would also be combined with other items (belonging to related neutral pairs) to form rearranged pairs. The same sample $(\mathrm{N}=10)$ of participants in prior relatedness ratings were recruited again and provided ratings for relatedness. The results confirmed our initial assignment of pairs, and showed that the relatedness scores of related pairs $[$ Mean $(\mathrm{SD})=6.67(1.23)]$ were significantly higher than that of unrelated pairs [Mean $(\mathrm{SD})=1.65(.57) ; t(299)=64.90, p<.001]$. Importantly, there was no difference for relatedness between the rearranged pairs and the original pairs [Unrelated pairs: $t(299)=1.07, p=$ 0.29; Related pairs: $t(299)=0.76, p=0.35]$.

A total of 400 picture pairs were encoded at the study phase (100 related negative pairs, 100 related neutral pairs, 100 unrelated negative pairs, 100 unrelated neutral pairs), with the remaining 200 pairs serving as new pairs during the test phase. At test, 200 intact pairs (the same pairs shown at study), 200 rearranged pairs (pictures belonging to different study pairs that were recombined together), and 200 new pairs were presented, with each condition containing 50 related negative, 50 related neutral, 50 unrelated negative, and 50 unrelated neutral pairs. Test pairs were counterbalanced across subjects, with every picture presented equally often as part of intact, rearranged, or new pairing.

Procedure

Participants were seated at a distance of $70 \mathrm{~cm}$ from a Dell monitor in an electrically shielded room. Picture pairs, with a visual area of $10^{\circ} \times 5^{\circ}$, were displayed (using Presentation by Neurobehavioral Systems, Inc) horizontally at the center of the monitor against a black background. A standard study- 
test paradigm was adopted, with the study phase followed by the test phase after a 10 min delay. Four self-paced breaks were provided during the study phase and during the test phase. Stimuli were presented pseudo-randomly to ensure that no more than three consecutive trials were from the same condition.

At study, each trial began with a gray fixation cross for $1000 \sim 1500 \mathrm{~ms}$, followed by the presentation of a picture pair for $2000 \mathrm{~ms}$, during which the participants were asked to memorize the pairs and perform a valence judgement task, namely, to judge which one of the two objects is more negative (Fig.1B). They were asked to press the 'left arrow' key on the keyboard if they thought that the left object was more negative, to press the 'right arrow' key if they thought that the right one was more negative, and to press the 'down arrow' key if they thought that the two objects had similar valence. Once the study phase was completed, a 10 min delay was provided. During this period, participants performed a distractor task of 3-digit backward counting for $5 \mathrm{~min}$ and then rested for five additional minutes.

At test, each trial began with a jittered fixation cross presented for $1000 \sim 1500 \mathrm{~ms}$, followed by the presentation of a picture pair for $2000 \mathrm{~ms}$. Participants were asked to indicate whether the pair is "intact", "rearranged", or "new" as accurately and as quickly as possible. Responses were provided via keyboard keys, counterbalanced across participants. Half of the participants made responses of "intact" and "rearranged" by pressing the key "F" and "G" with left hand, and of "new" by pressing the key "J" with right hand. The other half of participants responded "intact" and "rearranged" by pressing the key " $\mathrm{H}$ " and "J" with right hand, and "new" by pressing the key " $F$ " with left hand.

A study practice block of 12 trials was provided at the beginning of the experiment, prior to the 
study phase. An additional test practice block of 18 trials was provided prior to the test phase. During these practice sessions, the experimenter ascertained that the participants understood the task.

EEG recording and preprocessing

EEG was recorded using a 64-channel Neuroscan system and the electrode locations adhered to the extended international 10-20 system. The sampling rate was $500 \mathrm{~Hz}$ with a $0.05 \sim 100 \mathrm{~Hz}$ bandpass filter. Electrooculogram (EOG) was recorded by a pair of electrodes placed outside the outer canthi of each eye and one infraorbital to the left eye. The left mastoid was used as the reference site online, and EEG signals were re-referenced offline to the average of the left and right mastoid recordings. Impedance was kept below $5 \mathrm{k} \Omega$. EEG/EOG signals were filtered with a bandpass of $0.05 \sim 40 \mathrm{~Hz}$. EEG data from the test phase were segmented into $1100 \mathrm{~ms}$ epochs, corrected to the $100 \mathrm{~ms}$ pre-stimulus baseline. Epochs with voltage exceeding $\pm 75 \mu \mathrm{V}$ were excluded. Independent component analysis (ICA) was used to isolate and remove EOG blink artifacts. A minimum of 16 trials for each condition was required to ensure an acceptable signal-to-noise ratio, and four participants were excluded for failing to meet the minimal number of trials.

Mean numbers of related analyzed trials were 39 (intact), 26 (rearranged), and 38 (new) for negative pairs, and 37 (intact), 26 (rearranged), and 43 (new) for neutral pairs. Mean numbers of unrelated analyzed trials were 25 (intact), 30 (rearranged), and 37 (new) for negative pairs, and 27 (intact), 30 (rearranged), and 41 (new) for neutral pairs. 
Statistical analyses

Data were extracted for correct trials only (Donaldson \& Rugg, 1998; Paller et al., 2003). Repeated measures analyses of variance (ANOVAs) were conducted for inferential statistics, with GreenhouseGeisser correction for non-sphericity. Subsidiary analyses were performed using repeated measures ANOVAs or t-tests as appropriate. To control for Type I error rates, p-values were corrected for false discovery rate (FDR) with the Benjamini-Hochberg procedure (Benjamini \& Hochberg, 1995) at $p$ $<.05$. Because the current study focuses on mnemonic effects, only main effects and interactions that included the factor of response type are reported.

\section{Behavioral analyses}

Behavioral data of interest was associative Pr: a discrimination measure of old/new effects for associative memory, defined by subtracting false alarm rates for rearranged pairs from hit rates for intact pairs (Jäger et al., 2006; Snodgrass \& Corwin, 1988). This measure was used to dissociate potential response bias (e.g., for related pairs; Ahmad \& Hockley, 2014; Liu et al., 2019; Tibon et al., 2014) from a true memory advantage. Pr's were analyzed using a repeated measure analysis of variance (ANOVA) with relatedness (related or unrelated) and with valence (negative or neutral) as repeated factors, and with Pr score as the dependent measure. In addition, we analyzed accuracy rates (\% correct), using a repeated ANOVA with relatedness (related or unrelated), valence (negative or neutral) and response type (intact, rearranged, or new) as repeated factors, and accuracy rate as the dependent measure. 


\section{ERP analyses}

Both intact and rearranged pairs are comprised of studied items. However, while intact pairs further contain studied associative information, rearranged pairs contain novel associative information which was not presented at study. Therefore, the intact/rearranged effects, i.e., differences between ERPs associated with correct "intact" judgments vs. correct "rearranged" judgments, is indicative of associative recognition. Furthermore, both rearranged and new pairs do not contain studied associative information. However, while rearranged pairs contain studied items, new pairs contain novel items that were not presented during the study phase. Therefore, the rearranged/new effect, i.e., differences between ERPs associated with correct "rearranged" judgments vs. correct "new" judgments, is indicative of item recognition. Therefore, we focused on the comparison between intact and rearranged pairs to index associative memory, and on the comparison between rearranged and new pairs to index item memory (e.g., Li et al., 2017; Rhodes \& Donaldson, 2008; Zheng et al., 2015).

For the frontal and parietal memory effects, time segments and regions of interest were defined based on previous ERP studies (Bader et al., 2010; Han et al., 2018; Li et al., 2017, 2019; Rugg \& Curran, 2007; Speer \& Curran, 2007; Wolk et al., 2006; Zheng et al., 2016). Accordingly, two time windows, $300-550 \mathrm{~ms}$ and $550-800 \mathrm{~ms}$, were used to capture the frontal and parietal memory effects, respectively. Mean amplitudes for statistical analyses in these windows were obtained from frontal (collapsed across F3, Fz, and F4), central (collapsed across C3, Cz, and C4) and parietal (collapsed across P3, Pz, and P4) scalp locations (Han et al., 2018; Hou et al., 2013; Molinaro et al., 2011).

Repeated measures ANOVA was conducted separately for each time window. For associative memory, the ANOVAs included four within-subjects factors: relatedness (related or unrelated), valence 
(negative or neutral), response type (intact or rearranged), and location (frontal, central, or parietal). For item memory, the ANOVA was the same, but included different response types (rearranged or new).

\section{Results}

Behavioral results

\section{Associative $\mathrm{Pr}$}

Means and SDs for the various behavioral measures of Experiment 1 are shown in Table 1. The ANOVA for associative $\operatorname{Pr}$ (relatedness $\times$ valence) revealed a significant main effect of valence, $F(1$, $41)=24.28, p<.001, \eta_{\mathrm{p}}^{2}=0.37$ (greater for neutral vs. negative pairs), and of relatedness, $F(1,41)=$ $120.01, p<.001, \eta_{\mathrm{p}}^{2}=0.75$ (greater for related vs. unrelated pairs). The analysis further revealed a significant 2-way interaction between relatedness and valence, $F(1,41)=6.61, p=.014, \eta^{2} \mathrm{p}=0.14$, with lower associative Pr for negative pairs (vs. neutral) in the unrelated condition, $t(41)=5.38, p$ $<.001, \mathrm{~d}=0.83$, but not in the related condition, $t(41)=1.65, p=0.107, \mathrm{~d}=0.25$.

\section{Accuracy rates}

The ANOVA (relatedness $\times$ valence $\times$ response type) on accuracy rates revealed main effects of relatedness, $F(1,41)=148.19, p<.001, \eta^{2}{ }_{\mathrm{p}}=0.78$, valence, $F(1,41)=34.65, p<.001, \eta_{\mathrm{p}}^{2}=0.46$, with greater accuracy rates for related pairs (vs. unrelated), and for neutral pairs (vs. negative). A main effect also emerged for response type, $F(2,82)=104.41, p<.001, \eta_{\mathrm{p}}^{2}=0.72$, with greater accuracy rates for new pairs vs. intact, $t(41)=10.94, p<.001, \mathrm{~d}=1.69$, for intact pairs vs. rearranged, $t(41)=$ 4.22, $p<.001, \mathrm{~d}=2.14$, and for new pairs vs. rearranged: $t(41)=13.87, p<.001, \mathrm{~d}=0.65$. There was 
also a 2-way interaction between relatedness and response type, $F(1.63,66.69)=140.70, p<.001, \eta^{2} \mathrm{p}$ $=0.77$, a 2-way interaction between valence and response type, $F(2,82)=13.46, p=<.001, \eta^{2}{ }_{\mathrm{p}}=0.25$, a 2-way interaction between relatedness and valence, $F(1,41)=9.26, p=.004, \eta^{2}{ }_{\mathrm{p}}=0.18$, as well as a 3-way interaction between all three factors, $F(2,82)=6.68, p=.002, \eta^{2}{ }_{\mathrm{p}}=0.14$.

We decomposed the 3-way interaction using separate follow-up ANOVAs for each response type, with the factors of relatedness and valence. For intact pairs, this analysis revealed a main effect of relatedness, $F(1,41)=361.91, p<.001, \eta_{\mathrm{p}}^{2}=0.90$, and a 2 -way interaction between the two factors, $F$ $(1,41)=17.35, p<.001, \eta_{\mathrm{p}}^{2}=0.30$, resulting from lower accuracy rates for negative pairs (vs. neutral) in the unrelated condition, $t(41)=2.78, p=.008, \mathrm{~d}=0.43$, but greater accuracy rates for negative pairs (vs. neutral) in the related condition, $t(41)=2.31, p=.026, \mathrm{~d}=0.36$. For rearranged pairs, this analysis revealed a main effect of relatedness, $F(1,41)=22.70, p<.001, \eta^{2}{ }_{\mathrm{p}}=0.36$. For new pairs this analysis revealed main effects of relatedness, $F(1,41)=7.45, p=.009, \eta^{2}{ }_{\mathrm{p}}=0.15$, and valence, $F(1,41)=$ $77.81, p<.001, \eta_{\mathrm{p}}^{2}=0.66$

Taken together, the behavioral results depict the predicted emotional associative interference effect, indicated by reduced Pr and accuracy rates for negative vs. neutral pairs. Furthermore, this effect was attenuated by semantic relatedness, with greater emotional interference observed for unrelated vs. related pairs. 
Table 1. Distribution of participants' responses and Pr scores in Experiment 1

\begin{tabular}{|c|c|c|c|c|c|}
\hline \multirow[b]{2}{*}{ Test pair type } & \multirow[b]{2}{*}{ Response } & \multicolumn{2}{|c|}{ Related } & \multicolumn{2}{|c|}{ Unrelated } \\
\hline & & Negative & Neutral & Negative & Neutral \\
\hline \multicolumn{6}{|l|}{ Intact } \\
\hline & Intact & $0.81(0.08)$ & $0.77(0.11)$ & $0.51(0.12)$ & $0.55(0.14)$ \\
\hline & Rearranged & $0.13(0.06)$ & $0.13(0.07)$ & $0.40(0.11)$ & $0.34(0.11)$ \\
\hline & New & $0.06(0.05)$ & $0.09(0.07)$ & $0.09(0.07)$ & $0.10(0.07)$ \\
\hline \multicolumn{6}{|l|}{ Rearranged } \\
\hline & Intact & $0.37(0.13)$ & $0.30(0.12)$ & $0.28(0.11)$ & $0.21(0.13)$ \\
\hline & Rearranged & $0.53(0.13)$ & $0.55(0.14)$ & $0.60(0.13)$ & $0.64(0.15)$ \\
\hline & New & $0.09(0.07)$ & $0.15(0.08)$ & $0.11(0.07)$ & $0.15(0.09)$ \\
\hline \multicolumn{6}{|l|}{ New } \\
\hline & Intact & $0.05(0.05)$ & $0.04(0.05)$ & $0.03(0.03)$ & $0.02(0.03)$ \\
\hline & Rearranged & $0.15(0.07)$ & $0.06(0.06)$ & $0.19(0.11)$ & $0.10(0.07)$ \\
\hline & New & $0.79(0.12)$ & $0.89(0.10)$ & $0.77(0.13)$ & $0.87(0.10)$ \\
\hline Assoc $\mathrm{Pr}$ & & $0.44(0.15)$ & $0.47(0.16)$ & $0.22(0.15)$ & $0.34(0.17)$ \\
\hline
\end{tabular}


ERP results

300-550-ms window

Intact vs. Rearranged. In the early time window, the ANOVA conducted to test the associative memory effect (Figures 2A and 2B) revealed a 2-way interaction between valence and response type, $F$ $(1,41)=9.29, p=.004, \eta_{p}^{2}=0.19$. Decomposition of the interaction using paired t-tests at each level of valence, revealed a significant associative memory effect (more positive-going waveforms for intact vs. rearranged) for negative pairs, $t(41)=3.13, p=.003, \mathrm{~d}=0.48$, but not for neutral pairs $(p=0.29)$. Thus, in the early time-window, the associative memory effect emerged for negative pairs, regardless their relatedness, and had a widespread distribution.

Rearranged vs. New. For item memory (Figures 2A and 2C), the ANOVA revealed a 2-way interaction between response type and location, $F(1.38,56.65)=8.49, p=.002, \eta^{2}=0.17$, and a 3 way interaction between relatedness, valence, and response type, $F(1,41)=6.25, p=.017, \eta^{2}{ }_{p}=0.13$. To decompose the 3-way interaction a follow-up ANOVA with the factors of relatedness and response type was conducted separately for each valence. For negative pairs, this analysis revealed no main effects or interactions that included the response type factor $(p s>.05)$. A similar follow-up ANOVA for neutral pairs revealed a main effect of response type, $F(1,41)=6.48, p=.015, \eta_{\mathrm{p}}^{2}=0.14$. Thus, in the early time window, the item memory effect was reliably observed for neutral pairs, but not for negative pairs. 
550-800-ms window

Intact vs. Rearranged. In the late time window, the ANOVA for the associative memory effect (Figures 2A and 2B) only revealed a main effect of response type, $F(1,41)=38.84, p<.001, \eta^{2}{ }_{\mathrm{p}}=0.49$, suggesting that the late associative memory effect was similarly observed in all conditions, and in all locations.

Rearranged vs. New. For item memory (Figures 2A and 2C), the ANOVA revealed a main effect of response type, $F(1,41)=6.77, p=.013, \eta^{2}{ }_{\mathrm{p}}=0.14$, and a 3 -way interaction between relatedness, valence, and response type, $F(1,41)=9.12, p=.004, \eta^{2}{ }_{\mathrm{p}}=0.18$. Decomposition of the 3 -way interaction revealed that for negative pairs, there was an interaction between relatedness and response type, $F$ (1, $41)=6.98, p=.012, \eta^{2}=0.15$, depicting a reversed item memory effect (i.e., greater positivity for new vs. rearranged pairs) for related pairs, $t(41)=2.41, p=.021, \mathrm{~d}=0.37$, but not for unrelated pairs $(p$ $=.2$ ). For neutral pairs, a reversed item memory effect was observed, evident as a main effect of response type, $F(1,41)=7.01, p=.011, \eta^{2} \mathrm{p}=0.15$, with no interaction with relatedness. Thus, overall, in the late time window, a reversed item memory effect emerged in all experimental conditions, except for unrelated negative pairs. 


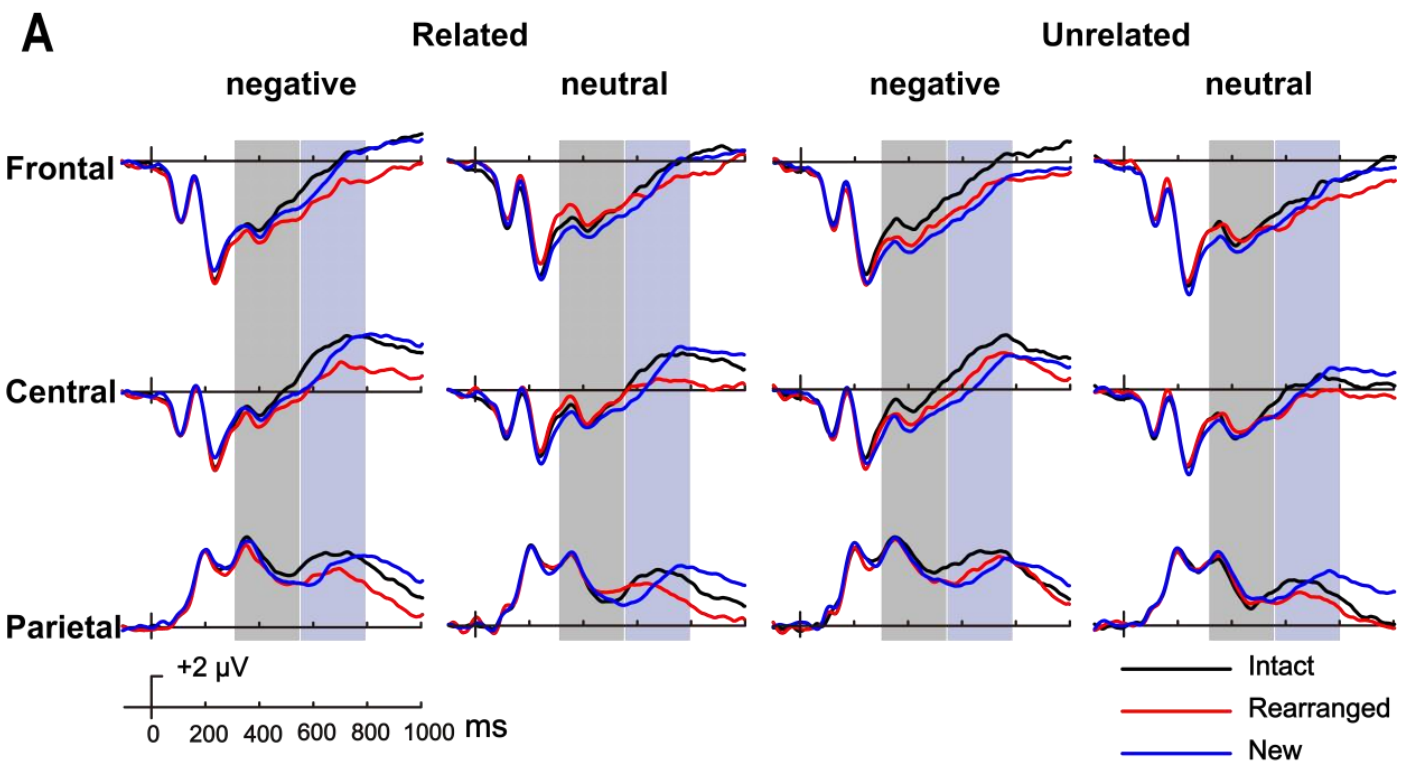

B

Intact vs. Rearranged

Related

$300-550 \mathrm{~ms}$

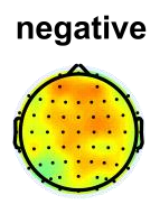

neutral

negative

Unrelated

$550-800 \mathrm{~ms}$
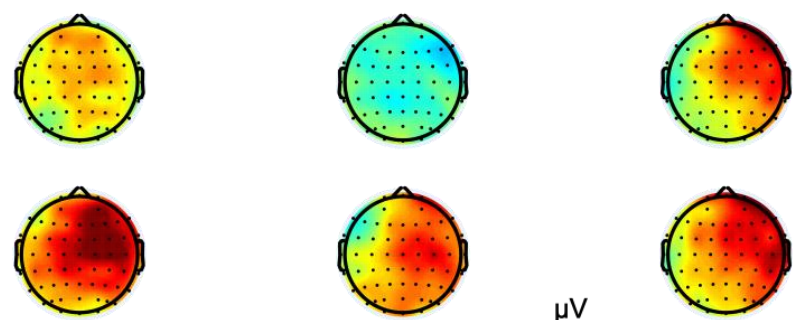

neutral
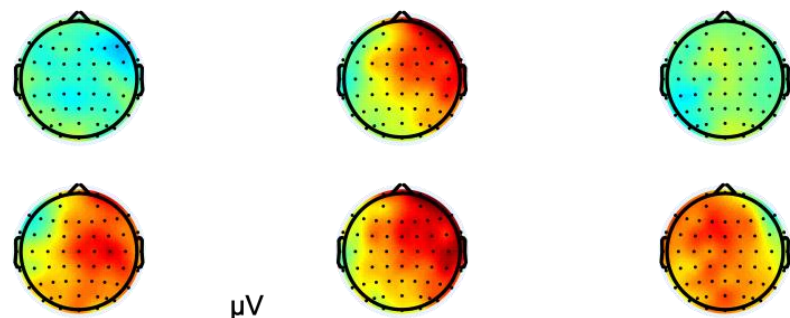

$\mu \mathrm{V}$

$+2$

C

Rearranged vs. New

Related

\section{Unrelated}

negative
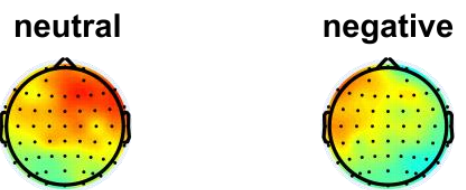

neutral

$300-550 \mathrm{~ms}$
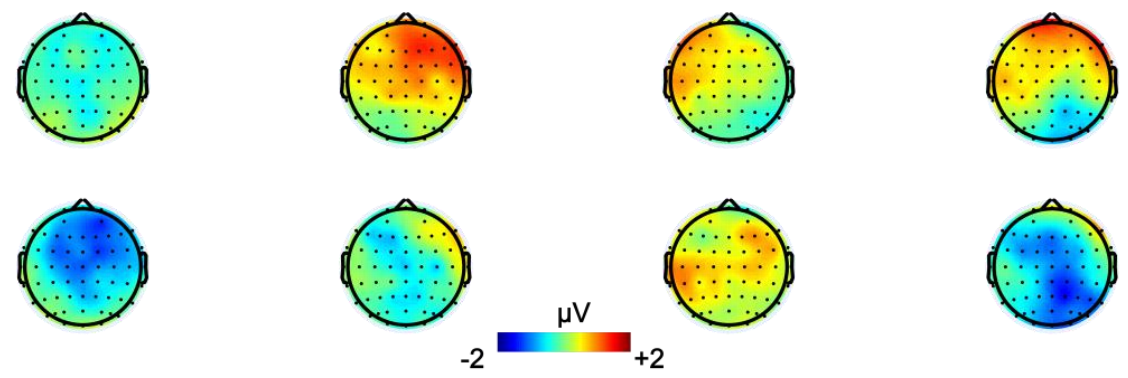

$\mu \mathrm{V}$

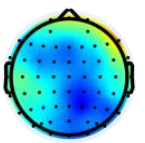

Figure 2. ERP results for Experiment 1. (A) Grand average ERP waveforms for intact responses (black), rearranged responses (red), and new responses (blue) in the four experimental conditions (relatedlunrelated $\times$ negativelneutral) at three scalp locations (F3, Fz, F4 collapsed as the frontal site; C3, Cz, C4 as the central site; P3, Pz, P4 as the parietal site). Shaded areas indicate time-windows used for the analyses of the early memory effects (300-550 ms, light grey) and the late memory effects (550-800 ms, dark gray). (B) Topographical maps of the associative memory effects (intact minus rearranged) in each time window. (C) Topographical maps of the item memory effects (rearranged minus new) in each time window. 


\section{Exploratory analysis: 800-1,000-ms window}

Following a visual inspection of the waveforms, we observed an additional associative memory effect between 800 and 1,000 ms, later than our two pre-defined time-windows. As an exploratory analysis, we investigated whether this effect is modulated by relatedness and/or valence. A repeated measures ANOVA with relatedness, valence, response type, and location as within-subject factors, revealed a main effect of response, $F(1,41)=32.36, p<.001, \eta^{2}{ }_{p}=0.44$, a 2 -way interaction between response type and location, $F(1.56,63.79)=8.02, p=.002, \eta_{p}^{2}=0.16$, as well as a 3 -way interaction between relatedness, response type and location, $F(1.50,61.59)=7.43, p=.003, \eta^{2} p=0.15$. A followup ANOVA with the factors of relatedness and response type was then conducted separately for each location. This analysis revealed a main effect of response type in all locations (all $p s<.001$ ). For parietal locations, this analysis also revealed an interaction between relatedness and response type, $F(1,41)=$ 4.52, $p=.040, \eta^{2}{ }_{p}=0.10$, depicting a significant associative memory effect for related pairs, $t(41)=$ $3.89, p<.001, d=0.60$, but not for unrelated pairs, $t(41)=1.39, p=0.18, d=0.21$. We consider these results in the General Discussion.

\section{Discussion}

In Experiment 1 we have observed an associative emotional interference effect, which was attenuated by semantic relatedness. Turning to the EEG data, semantic relatedness did not modulate the early associative memory effect, associated with familiarity, nor the late associative memory effect, associated with recollection. That is, the difference waveform for intact vs. rearranged pairs was similar for related and unrelated pairings. Interestingly, however, we have observed an emotional effect on 
associative memory: the early ERP component showed a frontal modulation of the associative memory effect for negative pairs (with greater frontal negativity for rearranged vs. intact pairings), suggesting unitizability of negative stimuli and the contribution of familiarity to their associative recognition. Nevertheless, associative emotional interference was only attenuated when semantically related pairs were retrieved, even though the modulation of the early ERP effect was similar for related and unrelated pairs. We return to this seemingly discrepancy between the behavioral and the ERP results in our General Discussion.

Although somewhat unpredicted, the modulation of the early ERP component for negative (but not neutral) pairs, adheres to the suggestion that emotionality serves as an organizing principle. Previous studies (Talmi \& Moscovitch, 2004; Talmi et al., 2007) proposed that the common finding of enhanced memory for emotional items can be due to a shared emotional context. In a series of experiments, they showed that the memory advantage for emotional items is eliminated when these are compared with categorized neutral items, suggesting that emotionality serves as an organizing principle for the items. This organization, along the dimension of emotional valence, possibly overshadowed the effect of semantic relatedness.

Indeed, in the current experiment, emotional content was explicitly probed (i.e., during study participants completed valence judgments for the stimuli), which might have promoted emotional processing and hinder processing of semantic relations. Notably, a growing number of evidence suggests that modulation of behavior or processing by emotional content is only triggered when emotions are relevant to the task (e.g., Engen et al., 2017; Huang et al., 2008; Pessoa, 2009; Stein et al., 2009; Wei et al., 2015). For example, Stein et al (2009) conducted the attentional blink task to estimate whether task relevance impacts prioritization. In this experiment, participants judged either the emotion 
(relevant condition) or gender (irrelevant condition) of two target facial stimuli depicting different expressions (fearful or neutral). Fearful faces (vs. neutral) induced a stronger attentional blink, but only in the relevant condition. This demonstrates that the processing advantage of emotional stimuli depends on the relevance of emotion to the task. Similarly, in the context of the current task, situating emotion as task-relevant might have promoted binding along this dimension. In other words, instead of creating unitized links for semantically related (vs. unrelated) items, such links were created for negative (vs. neutral) ones. Experiment 2 was designed to address this possibility.

\section{Experiment 2}

In Experiment 2, we sought to investigate the effect of semantic relatedness on associative recognition of emotional stimuli, when emotions are incidental to the task. To this end, Experiment 2 employed the same behavioral and EEG measures as in Experiment 1, but the study task differed. Namely, rather than valence judgment, in Experiment 2 participants were asked to perform a familiarity judgment.

We predicted an overall behavioral pattern similar to that obtained for Experiment 1, that is, greater associative emotional interference effect for related than for unrelated pairs. Nevertheless, because in Experiment 2 emotion is irrelevant to the encoding task, we expected that this difference would be attenuated. We further rationalized that, with emotion being incidental to the task, unitized links and elaborative encoding for semantically related (vs. unrelated) items would be readily achieved. We therefore predicted that both the early and the late associative memory ERP effects would be greater for related than unrelated stimuli. 


\section{Method}

Participants

Forty-seven healthy, right-handed native Chinese speakers ( 32 females; mean age $22.1 \pm 1.9$ years) from Capital Normal University participated, with the same characteristics as those who participated in Experiment 1, participated in Experiment 2. Data from seven participants were discarded, due to insufficient number of artifact-free ERP trials in one or more experimental conditions $(\mathrm{N}<16)$. Our final sample therefore included 40 participants ( 27 females; mean age $21.9 \pm 2.0$ years).

Procedure, recording, and analyses

Stimuli were the same as in Experiment 1. The procedure was identical to that of Experiment 1, except that participants were asked to perform a familiarity judgement task at study, namely, to judge which one of the two objects presented in each trial was more familiar. They were asked to press the 'left arrow' key on the keyboard if they thought that the left object was more familiar, to press the 'right arrow' key if they thought that the right one was more familiar, and to press the 'down arrow' key if they thought that the two objects do not differ in their familiarity.

EEG recording and preprocessing were the same as in Experiment 1. Mean numbers of related analyzed trials were 38 (intact), 26 (rearranged), and 39 (new) for negative pairs, and 40 (intact), 29 (rearranged), and 43 (new) for neutral pairs. Mean numbers of unrelated analyzed trials were 23 (intact), 29 (rearranged), and 36 (new) for negative pairs, and 28 (intact), 31 (rearranged), and 42 (new) for neutral pairs. Statistical analyses were the same as for Experiment 1. 


\section{Results}

Behavioral results

\section{Associative Pr}

Means and SDs for the various behavioral measures of Experiment 2 are shown in Table 2. The ANOVA for associative Pr revealed main effects of valence, $F(1,39)=40.31, p<.001, \eta^{2}{ }_{\mathrm{p}}=0.51$, and relatedness, $F(1,39)=220.81, p<.001, \eta_{\mathrm{p}}^{2}=0.85$, with greater Pr scores for neutral pairs (vs. negative), and for related pairs (vs. unrelated).

\section{Accuracy rates}

For accuracy rates, the analysis revealed main effects of relatedness, $F(1,39)=198.92, p<.001$, $\eta_{\mathrm{p}}^{2}=0.84$, and valence, $F(1,39)=97.18, p<.001, \eta_{\mathrm{p}}^{2}=0.71$, with greater accuracy rates for related pairs (vs. unrelated), and for neutral pairs (vs. negative). A main effect was also revealed for response type, $F(2,78)=111.76, p<.001, \eta_{\mathrm{p}}^{2}=0.74$, with greater accuracy rates for new vs. intact pairs, $t(39)$ $=10.69, p<.001, \mathrm{~d}=1.69$, and vs. rearranged pairs, $t(39)=13.82, p<.001, \mathrm{~d}=2.19$, and for intact vs. rearranged pairs, $t(39)=4.47, p<.001, \mathrm{~d}=0.70$. There were also 2-way interactions between relatedness and response type, $F(1.51,59.00)=151.17, p<.001, \eta^{2}{ }_{\mathrm{p}}=0.80$, between valence and response type, $F(2,78)=9.52, p<.001, \eta^{2} \mathrm{p}=0.20$, and between relatedness and valence, $F(1,39)=$ $6.84, p=.013, \eta_{\mathrm{p}}^{2}=0.15$. Finally, the analysis revealed a significant 3 -way interaction between all 3 factors, $F(2,78)=8.52, p<.001, \eta^{2}{ }_{\mathrm{p}}=0.18$.

Decomposition of the 3-way interaction, separately for each response type, revealed that for intact pairs and new pairs, there was a main effects of relatedness [intact: $F(1,39)=370.64, p<.001, \eta^{2}{ }_{\mathrm{p}}=$ 
0.91; new: $\left.F(1,39)=25.88, p<.001, \eta_{\mathrm{p}}^{2}=0.40\right]$, and valence [intact: $F(1,39)=22.64, p<.001, \eta^{2} \mathrm{p}$ $=0.37$; new: $\left.F(1,39)=107.67, p<.001, \eta^{2}{ }_{\mathrm{p}}=0.73\right]$, as well as a 2 -way interaction between the two factors [intact: $F(1,39)=12.67, p=.001, \eta_{\mathrm{p}}^{2}=0.25$; new: $\left.F(1,39)=7.64, p=.009, \eta_{\mathrm{p}}^{2}=0.16\right]$. Decomposition of the interaction between relatedness and valence revealed that even though the difference in accuracy rates between neutral and negative pairs emerged for both related and unrelated pairs, it was greater in the latter [intact: $t_{\text {related }}(39)=2.35, p=.024, \mathrm{~d}=0.37 ; t_{\text {unrelated }}(39)=5.26, p$ $<.001, \mathrm{~d}=0.83$; new: $\left.t_{\text {related }}(39)=7.57, p<.001, \mathrm{~d}=1.20 ; t_{\text {unrelated }}(39)=9.97, p<.001, \mathrm{~d}=1.58\right]$. For rearranged pairs, this analysis revealed main effects of relatedness, $F(1,39)=12.88, p=.001, \eta^{2}{ }_{\mathrm{p}}=$ 0.25 , and valence, $F(1,39)=6.59, p=.014, \eta^{2}=0.15$.

The results of Experiment 2 show a similar pattern of attenuated emotional interference effect for related vs. unrelated pairs, which we observed in Experiment 1. However, in contrast with Experiment 1, in which this pattern was observed both in the unbiased associative Pr measure and intact responses, in Experiment 2 this pattern was observed for intact (and new) pairs, but not for Pr scores. 
Table 2. Distribution of participants' responses and Pr scores in Experiment 2

\begin{tabular}{|c|c|c|c|c|c|}
\hline \multirow[b]{2}{*}{ Test pair type } & \multirow[b]{2}{*}{ Response } & \multicolumn{2}{|c|}{ Related } & \multicolumn{2}{|c|}{ Unrelated } \\
\hline & & Negative & Neutral & Negative & Neutral \\
\hline \multicolumn{6}{|l|}{ Intact } \\
\hline & Intact & $0.79(0.08)$ & $0.83(0.10)$ & $0.48(0.12)$ & $0.59(0.14)$ \\
\hline & Rearranged & $0.16(0.06)$ & $0.11(0.05)$ & $0.43(0.10)$ & $0.34(0.10)$ \\
\hline & New & $0.06(0.05)$ & $0.06(0.07)$ & $0.09(0.07)$ & $0.07(0.07)$ \\
\hline
\end{tabular}

Rearranged

$\begin{array}{ccccc}\text { Intact } & 0.36(0.12) & 0.29(0.11) & 0.26(0.09) & 0.27(0.12) \\ \text { Rearranged } & 0.54(0.12) & 0.60(0.13) & 0.62(0.12) & 0.64(0.14) \\ \text { New } & 0.10(0.05) & 0.11(0.09) & 0.12(0.07) & 0.09(0.06)\end{array}$

New

\begin{tabular}{lccccc} 
& Intact & $0.05(0.06)$ & $0.02(0.04)$ & $0.04(0.04)$ & $0.01(0.02)$ \\
& Rearranged & $0.15(0.08)$ & $0.07(0.05)$ & $0.21(0.12)$ & $0.11(0.08)$ \\
& New & $0.80(0.12)$ & $0.90(0.08)$ & $0.74(0.14)$ & $0.89(0.10)$ \\
\hline \multirow{2}{*}{ Assoc Pr } & & $0.43(0.13)$ & $0.54(0.15)$ & $0.22(0.12)$ & $0.31(0.18)$ \\
\hline
\end{tabular}

Note: standard deviations are shown in parenthesis

ERP results

300-550-ms window

Intact vs. Rearranged. For associative memory (Figures 3A and 3B), the analysis revealed a main effect of response type, with more positive-going waveforms for intact pairs (vs. rearranged), $F(1,39)$ $=4.67, p=.037, \eta_{\mathrm{p}}^{2}=0.11$, and 2-way interaction between relatedness and response type, $F(1,39)=$ $5.09, p=.030, \eta_{\mathrm{p}}^{2}=0.12$. Decomposition of the interaction revealed a significant associative memory 
effect for related pairs, $t(39)=3.08, p=.004, \mathrm{~d}=0.49$, but not for unrelated pairs $(p=0.81)$. Thus, in the early time-window, the associative memory effect emerged for related pairs, regardless their valence, and had a widespread distribution.

Rearranged vs. New. For item memory, the analysis revealed a main effect of response type, with more positive-going waveforms for rearranged pairs (vs. new), $F(1,39)=4.55, p=.039, \eta_{\mathrm{p}}^{2}=0.10,2$ way interaction between relatedness and response type, $F(1,39)=4.17, p=.048, \eta_{\mathrm{p}}^{2}=0.10$, a 2 -way interaction between response type and location, $F(1.25,48.55)=10.76, p=.001, \eta^{2} \mathrm{p}=0.22$, and a 3way interaction between relatedness, valence, and response type, $F(1,39)=9.26, p=.004, \eta_{\mathrm{p}}^{2}=0.19$.

To decompose the 3-way interaction, a follow-up ANOVA with the factors of relatedness and response type was then conducted separately for each valence. For negative pairs, this analysis revealed an interaction between relatedness and response type, $F(1,39)=12.57, p=.001, \eta_{\mathrm{p}}^{2}=0.24$, with a significant item memory effect (rearranged vs. new) for unrelated pairs, $t(39)=3.48, p=.001, \mathrm{~d}=0.55$, but not for related pairs $(p=.36)$. A similar follow-up ANOVA for neutral pairs revealed no main effects or interactions $(p s>.05)$.

550-800-ms window

Intact vs. Rearranged. In the late time window, the analysis of the associative memory effect revealed a main effect of response type, $F(1,39)=38.49, p<.001, \eta^{2}{ }_{p}=0.50$, a 2 -way interaction between relatedness and response type, $F(1,39)=10.23, p=.003, \eta^{2}=0.21$, a 2-way interaction between valence and response type, $F(1,39)=6.92, p=.012, \eta^{2}=0.15$, and a 3 -way interaction between relatedness, valence, and response type, $F(1,39)=8.37, p=.006, \eta^{2}{ }_{p}=0.18$. 
To decompose the 3-way interaction, we conducted follow-up ANOVAs with relatedness, and response type as within-subject factors, separately for each valence. For negative pairs, this analysis revealed a main effect of response type, $F(1,39)=11.46, p=.002, \eta^{2}{ }_{p}=0.23$, and a significant 2-way interaction between relatedness and response type, $F(1,39)=17.12, p<.001, \eta^{2}{ }_{p}=0.31$, resulting from an associative memory effect for related pairs, $t(39)=4.74, p<.001, \mathrm{~d}=0.75$, but not for unrelated pairs $(p=.82)$. A similar follow-up ANOVA for neutral pairs revealed a main effect for response type, $F(1,39)=42.93, p<.001, \eta^{2} p=0.52$, but no interaction between the factors.

Rearranged vs. New. For item-memory, the analysis revealed 2-way interactions between relatedness and response type, $F(1,39)=6.18, p=.017, \eta_{p}^{2}=0.14$, and between valence and response type, $F(1,39)=14.14, p=.001, \eta_{p}^{2}=0.27$. Finally, the analysis revealed a 3-way interaction between relatedness, valence, and response type, $F(1,39)=4.51, p=.040, \eta^{2}{ }_{\mathrm{p}}=0.10$.

To decompose the 3-way interaction, we conducted follow-up ANOVAs with relatedness and response type as within-subject factors, separately for each valence. For negative pairs, this analysis revealed a 2-way interaction between relatedness and response type, $F(1,39)=12.81, p=.001, \eta^{2}{ }_{p}=$ 0.25 , resulting from an item memory effect (rearranged vs. new) for unrelated pairs, $t(39)=3.45, p$ $=.001, \mathrm{~d}=0.54$ but not for related pairs $(p=.24)$. A similar follow-up analysis for neutral pairs revealed a main effect for response type, $F(1,39)=14.19, p=.001, \eta^{2}{ }_{p}=0.27$, with more positive-going waveforms for new vs. rearranged pairs (a "reversed" item memory effect), regardless their relatedness. Thus overall, at the late time window, an item memory effect emerged for unrelated negative pairs, and a reversed item memory effect (of reversed polarity) emerged for related and unrelated neutral pairs. 


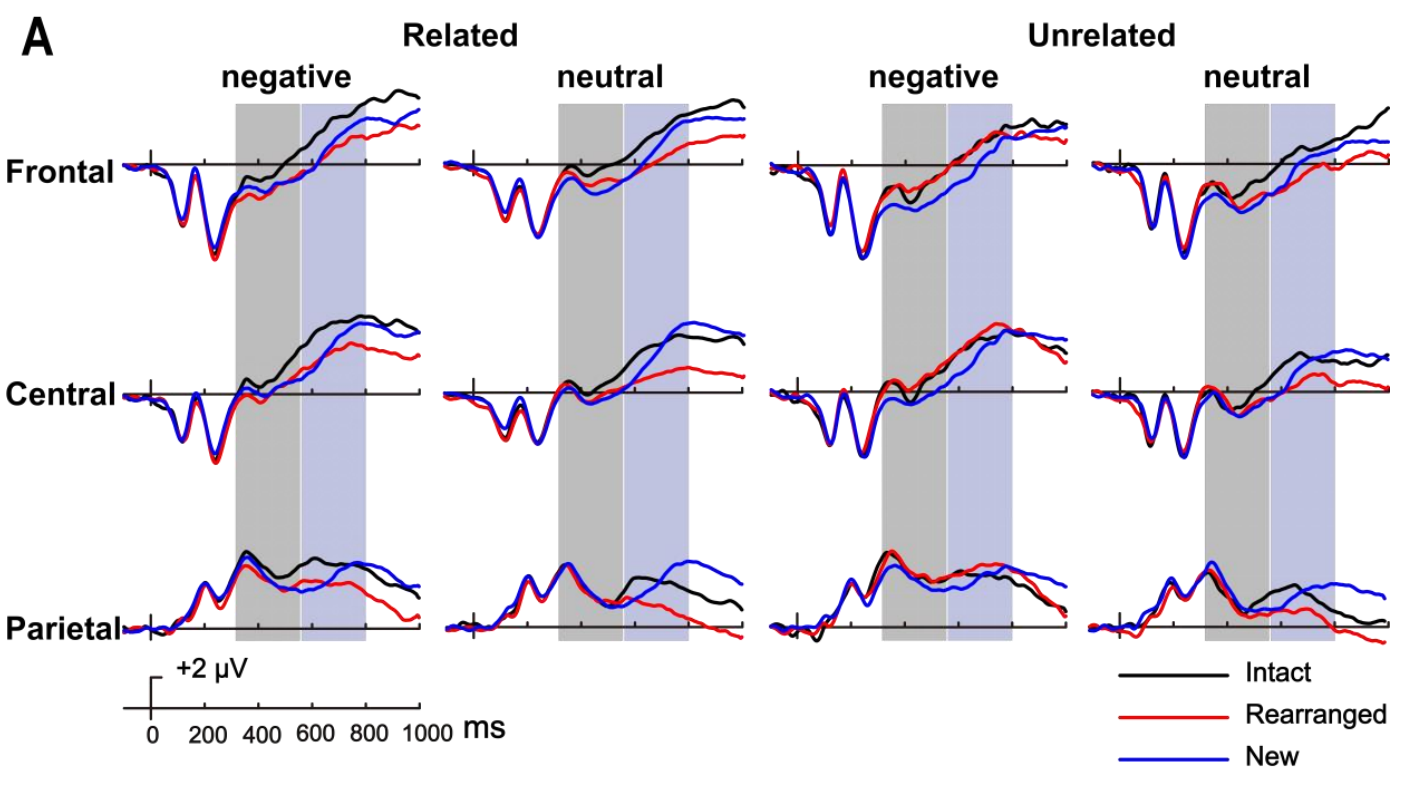

B

Intact vs. Rearranged

Related

Unrelated

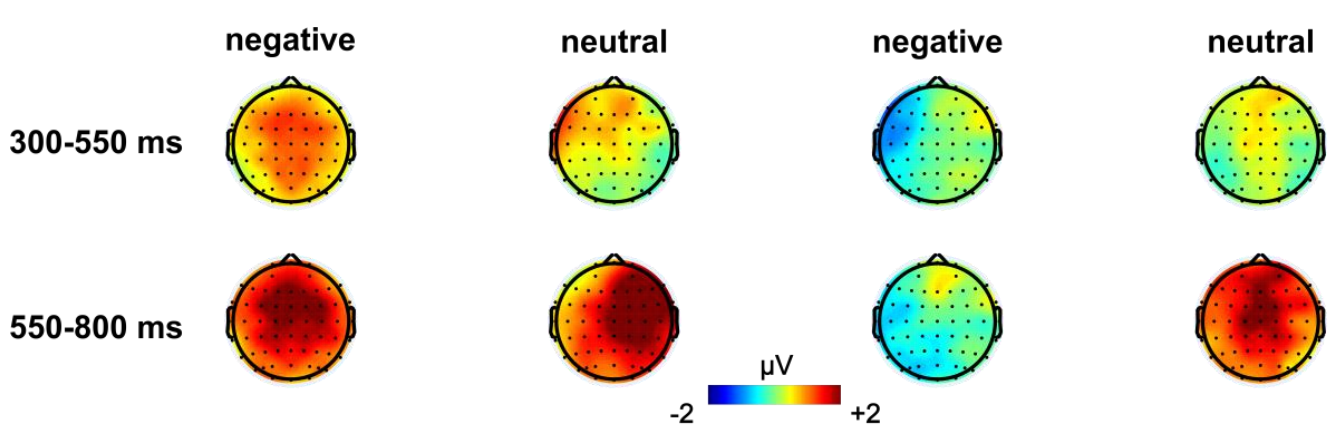

C

Rearranged vs. New

Related
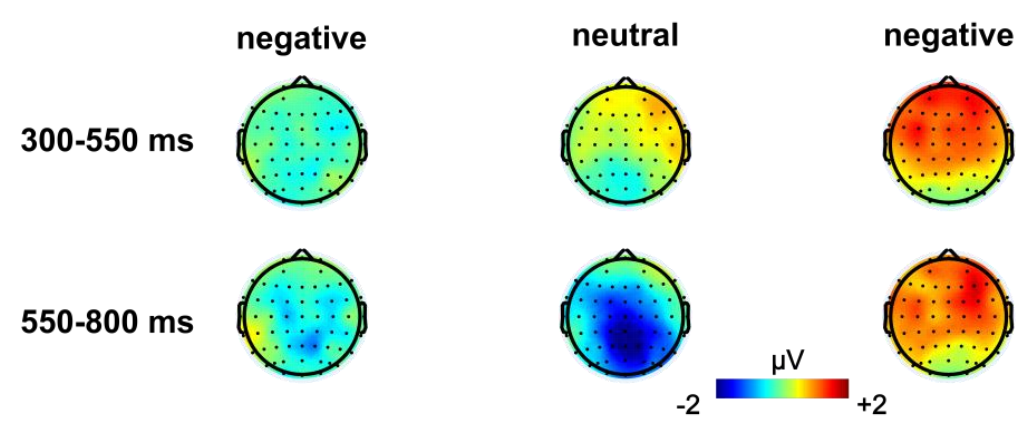

Unrelated

neutral
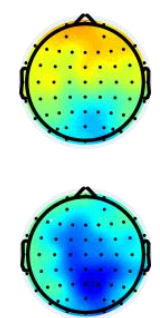

Figure 3. ERP results for Experiment 2. (A) Grand average ERP waveforms for intact responses (black), rearranged responses (red), and new responses (blue) in the four conditions at three scalp locations. Shaded areas indicate time-windows used for the analysis of the early and late memory effects. (B) Topographical maps of the associative memory effects in each time window. (C) Topographical maps of the item memory effects in each time window. 
Exploratory analysis: 800-1,000-ms window

Once again, visual inspection of the waveforms suggested that an associative memory effect is also apparent between 800 and 1,000 ms. A repeated measures ANOVA at this time window revealed a main effect of response type, $F(1,39)=30.55, p<.001, \eta^{2}{ }_{p}=0.44$, a 2 -way interaction between relatedness and response type, $F(1,39)=11.13, p=.002, \eta^{2}{ }_{p}=0.22$, a 2 -way interaction between response type and location, $F(1.61,62.77)=7.86, p=.002, \eta^{2}{ }_{p}=0.17$, and a 3 -way interaction between relatedness, response type, and location, $F(1.64,63.78)=3.69, p=.039, \eta^{2}{ }^{2}=0.09$. A follow-up ANOVA with the factors of relatedness and response type was then conducted separately for each location. This analysis revealed main effects of response type in all locations (all $p \mathrm{~s}<.001$ ), as well as a 2-way interaction between relatedness and response type in central and parietal locations [central: $F(1,39)=9.95, p$ $=.003, \eta_{p}^{2}=0.20$; parietal: $\left.F(1,39)=13.57, p=.001, \eta_{p}^{2}=0.26\right]$. Decomposition of this interaction revealed that in central locations, the associative memory effect was greater for related pairs, $t(39)=$ 5.23, $p<.001, \mathrm{~d}=0.83$, vs. unrelated pairs $t(39)=2.61, p=.013, \mathrm{~d}=0.41$. In parietal locations the effect was only observed for related pairs, $t(39)=5.44, p<.001, \mathrm{~d}=0.86$, but no for unrelated pair, $t$ $(39)=1.34, p=.19, \mathrm{~d}=0.21$.

\section{Discussion}

In Experiment 2, the attenuation of the associative emotional interference effect for related information was apparent in the accuracy rates (specifically in the intact condition, which contains associative information) but not in associative Pr scores. This indicates that participants were more likely to classify negative pairs as intact if they were semantically related, but this tendency occurred 
for both intact and rearranged pairs. We come back to this in the General Discussion below.

As for the ERP data, the results agree with previous studies (e.g., Ahmad \& Hockley, 2014; Kriukova et al., 2013; Li et al., 2019; Rhodes \& Donaldson, 2008; Tibon et al., 2014), demonstrating modulation of the early associative memory effect by semantic relatedness. Namely, the early difference between intact and rearranged pairs was only significant when stimuli were semantically related (regardless their valence). The late associative memory effect was more generally distributed, and was only absent for unrelated negative pairs. While this absence might seem puzzling at first sight, a closer look at the behavioral results suggests that this is the only experimental condition (across both experiments) for which the probability to correctly classify intact items (47\%) was highly similar to the probability to classify them as rearranged pairs (43\%). This suggests that this condition, for which emotional associative interference is present (due to negative valence), and is not attenuated by semantic relations, recollective processes are impeded, as reflected in the late ERP component. Yet, it remains unclear why the same pattern was not observed in Experiment 1.

\section{General Discussion}

The present study utilized an associative recognition paradigm, across two experiments, to investigate how pre-existing relations, the valence of the stimuli, and their interaction, affect our ability to retrieve associative information. Both experiments conformed to the same procedure, aside from the instructions given during the study phase. Namely, in Experiment 1 participants were asked to compare the valence of object pairs, whereas in Experiment 2, participants were asked to compare their familiarity. 
In both experiments, an associative emotional interference effect had emerged, with reduced associative memory for negative pairs. This finding agrees with previous studies, showing that emotion can impair associative memory (Madan et al., 2012; Mao et al., 2015; Mather \& Knight, 2008; Pierce \& Kensinger, 2011; Rimmele et al., 2011). Importantly, our results further suggest that this associative emotional interference effect can be attenuated under different circumstances. In particular, when valence is salient (as in the case of Experiment 1, where the task requires valence judgment) semantic associative relations can attenuate this associative emotional interference effect. Interestingly, in Experiment 2, negative related pairs were more likely to be classified as "intact" compared to negative unrelated pairs (as indicated by their accuracy scores). This tendency, however, similarly occurred for both intact and rearranged pairs (as indicated by the unbiased associative Pr measure), and thus, in the case of Experiment 2, does not reflect better memory but rather a response bias. Taken together, these results agree with previous research, showing that emotions can enhance processing, but only when they are relevant to the task (e.g., Engen et al., 2017; Huang et al., 2008; Pessoa, 2009; Stein et al., 2009; Wei et al., 2015). In the current case, during encoding, processing of semantic relations was enhanced for negative pairs, leading to better binding and improved memory trace. Nevertheless, this mnemonic advantage only occurred when emotions were task-relevant (i.e., in Experiment 1).

Turning to the ERP data, our results revealed that the modulation of the early associative memory effect was task dependent. Specifically, in Experiment 1, where emotions were relevant to encoding, the early ERP effect showed greater frontal negativity for intact vs. rearranged negative pairs, regardless their semantic relations. In contrast, in Experiment 2, where familiarity (and not valence) was probed during encoding, this modulation only occurred for related (but not for unrelated) pairs, regardless their valence. These results provide evidence for the suggestion that emotionality triggered by stimulus' 
valence can serve as an organizing principle that can bind stimuli together via shared context (Riberto et al., 2019; Talmi \& Moscovitz, 2004). Therefore, pairs of emotional stimuli might be more easily unitized compared to non-emotional ones. The results of Experiment 1 support this idea. Namely, in this experiment, the early associative memory effect - the putative electrophysiological correlate of familiarity—showed a modulation for negative pairs, indicating that familiarity was readily available for these pairs.

Interestingly, this valence-based modulation of the early effect was not observed in Experiment 2, in which valence was not probed during encoding, suggesting that emotionality is more likely to serve as an organizing principle when valence is task relevant (or salient). When valence is not relevant to the task, familiarity signals can be elicited when the stimuli comprising the pair are semantically related, as apparent in Experiment 2. Indeed, previous studies have shown that semantic relations can promote unitization, which further enhances familiarity-based associative recognition (e.g., Ahmad \& Hockley, 2014; Kriukova el al., 2013; Li et al., 2019; Rhodes \& Donaldson, 2008; Tibon et al., 2014). Arguably, when valence is irrelevant, semantic relatedness "pops-out" as a salient organizing principle, and enables unitization along this dimension. Taken together, the modulation of the early associative effect observed in the current study, suggests that both semantic relationships and emotional context can promote unitization which can subsequently trigger familiarity-based retrieval.

Unlike the selective modulation of the early associative memory effects, the modulation of the late effect was apparent more generally across the various experimental conditions (albeit, as noted above, not for unrelated negative pairs in Experiment 2). Although this later effect was rather broadly distributed and lacked the pronounced parietal maxima often associated with the recollection-related late positive component (Wilding \& Ranganath, 2011; Rugg \& Curran, 2007), retrieval-related 
modulations with anterior/central topographic distribution are commonly reported in associative recognition ERP studies (e.g., Bader et al., 2010; Han et al., 2018; Kriukova et al., 2013; Mollison \& Curran, 2012; Rhodes \& Donaldson, 2007, 2008; Tibon, Ben-Zvi, \& Levy, 2014; Zheng, Li, Xiao, Broster, \& Jiang, 2015) and are interpreted as reflecting recollective processes. Our findings suggest that recollective processes were readily available for the various stimuli, regardless their valence or their semantic relations.

One aspect of the associative memory effects that warrants further attention is the correspondence between the behavioral results and the ERP data. Specifically, in Experiment 1, associative emotional interference was only attenuated when semantically related pairs were retrieved, even though the modulation of the early ERP effect was similar for related and unrelated pairs. We speculate that the production of early mnemonic signals (as indicated in the ERPs) would only affect behavior if these signals are considered diagnostic or trusted. For semantically related pairs, the emotional context adjoins the semantic one (e.g., for gun:bullets pair: negative feeling due to the shooting gun), producing a trustworthy mnemonic signal that attenuates associative emotional interference. In contrast, for stimuli that lack semantic relations, the early signal is cognitively attributed to more general sources (e.g., that the stimuli were experienced together during the experimental session, rather than within specific pairing). Therefore, these signals are not designated as trustworthy, and do not produce the same behavioral change. This idea coincide with a recent proposal by Bastin et al (2019) which posits that an attribution system modulates the use of memory traces as a function of expectancies, task context, and goals, leading to subjective experiences and explicit judgments. In the current case, associative familiarity signals might have been attributed to an alternative source (e.g., such as processing fluency or global familiarity) and were discarded in the face of explicit judgments. 
Our exploratory analysis further revealed an additional associative memory effect, which was widespread at a later time window ranging from $800-1,000$ ms post stimulus onset. At parietal locations, this effect was further modulated by semantic relatedness, and thus corresponds to the behavioral advantage observed for related (vs. unrelated) pairs. Parietal regions had been implemented in sustained recollective processes that track the period over which recollected information is maintained (e.g., Vilberg \& Rugg, 2008; Vilberg \& Rugg, 2012; Vilberg \& Rugg, 2013; see also Humphreys et al., 2021, for a unifying cross-domain account). The elaborated encoding afforded by preexisting semantic relations might be gradually elicited, and therefore reflected in a later time window than our predefined one. We thus suspect that this late modulation reflects the continuous involvement of recollective processes (that is, an extension of the activation observed in the previous time window).

In addition to these associative memory effects, our study showed task-depended modulation of the early rearranged/new effect, which is indicative of item familiarity. Specifically, in Experiment 1, the early rearranges/new effect was greater for neutral (vs. negative) pairs, regardless their semantic relations. In Experiment 2, it was evident for negative unrelated pairs. It is interesting to note that, to some degree, these item memory effects mirror the early associative memory effects, which were obtained for negative pairs in Experiment 1, and for related pairs in Experiment 2. This pattern might indirectly support the suggestion that a shared emotional context and pre-existing semantic relations can promote unitization. It has been suggested that once multiple items have been unitized to create a single representation, only the unitized whole, and not the constituent elements, is readily available for familiarity at retrieval. As a consequence, unitization should entail measurable "costs" on item familiarity (Liu et al., 2020; Mayes et al., 2007; Pilgrim et al., 2012). Such costs are evident in our current data, where item-related modulation of the early ERP component was greater for non-unitizable 
pairs. This suggestion warrants further investigation in future studies.

In the late time window, greater positivity for new pairs (at a magnitude that is equivalent or greater than that of the intact pairs) was observed for related pairs and for unrelated-neutral pairs in Experiment 1, and for neutral pairs in Experiment 2. This somewhat unexpected pattern of intact $=$ new $>$ rearranged seems to be inconsistent with some previous reports of decreased positivity for new vs. intact/rearranged pairs (e.g., Bader et al., 2010; Greve et al., 2007; Kriukova et al., 2013; Mollison \& Curran, 2012; Opitz, 2010; Rhodes \& Donaldson, 2007, 2008). Nonetheless, it is comparable with other previous findings. For example, Tibon, Gronau, Scheuplein, Mecklinger, \& Levy (2014) found a pattern of intact = new > rearranged for unrelated pairs in the late time window; Lu, Liu, Wang, \& Guo (2020) found a similar ERP pattern for word-picture pairs in an interactive imagery condition; Addante, Ranganath, \& Yonelinas (2012) showed that the late parietal ERP was less positive for low confidence item recognition accompanied by accurate source recognition than for correctly rejected foils. One possibility is that the neural response that we have observed at the late time window reflects a combination of multiple ERP components with overlapping durations. In particular, the late posterior negativity component (LPN) tends to overlap with the traditional late old/new effect, and is of opposite polarity (for a review see Mecklinger et al., 2016). This component is often linked to reconstructive processing, evaluation of retrieval outcomes, and/or task difficulty, although the precise functional role of this old/new difference remains a matter of debate (see, for example, Friedman et al., 2005; Johansson \& Mecklinger, 2003; Mecklinger et al., 2016; Sommer et al., 2018; but also recent contradicting results by Park \& Donaldson, 2019). In the case of our study, we note that it remains unclear why certain experimental conditions, but not others, would elicit this effect.

In the current study, the distinction between the two processes supporting recognition memory- 
familiarity and recollection — relies mainly on the temporal distribution, and to some extent also on the spatial distribution, of the early and late intact/rearranged ERP effects. Like many other studies (e.g., Bader et al., 2010; Guillaume \& Etienne, 2015; Jäger et al., 2006; Kamp et al., 2016; Rhodes \& Donaldson, 2008; Tibon et al., 2014; Zheng et al., 2015, to list just a few), we link the early frontal negativity with familiarity, and the late positivity with recollection. While this type of reverse inference has its limitations (Poldrack, 2006), our interpretation builds on decades of intensive research that strongly associates these ERP components with the particular memory processes reported here (reviewed by Mecklinger, 2000; Rugg \& Curran, 2007; Wilding \& Ranganath, 2011). We do acknowledge that in the current study, the early ERP component might reflect processing fluency instead of (or possibly together with) familiarity (see Paller et al., 2007 for discussion). In any event, however, even if the links made in our study between electrophysiological measures and specific recognition processes are not entirely conclusive (though strongly suggestive), our data clearly point to a neural distinction, whereby the contribution of the early ERP effect to associative recognition is limited to certain experimental conditions, but the contribution of the late ERP effect is more widely available across different types of associative information.

One caveat of the current study, is that negative stimuli were also highly arousing (compared to neutral, see Stimuli section above). Therefore, one potential difficulty in interpretation of the present findings is that valence effects cannot be distinguished from arousal effects, even though prior studies suggest that these might rely on distinct neural process (e.g., Kensinger \& Corkin, 2004). Furthermore, the current study only included negative and neutral stimuli, precluding any conclusions regarding general emotional effects, or distinction between different kinds of valence. In addition, the study did not include any additional emotional measures, such as mood assessment or assessment of anxiety/stress 
symptoms, to be used as covariates in statistical analyses. Future studies are thus required in order to generalize our conclusions further.

In summary, the current study shows that when items share a context during their encoding, be that semantic or emotional, their associative retrieval can provoke an early neural modulation, arguably indicative of familiarity-based retrieval, which accompanies recollection. This modulation further depends on the way information was encoded: when valence is relevant to encoding, it acts as a salient organizing principle that triggers early retrieval processes. But when valence is not relevant, other relations (in our case, semantic) become salient and serve to organize information. Moreover, we propose that the neural modulation only relates to behavioral change when the signals are being interpreted as trustworthy, particularly, when pre-existing semantic relations between the various pieces of information are present. This suggests that in real-life situations, where emotional information is often highly relevant and semantically meaningful, activation of early mnemonic signals can be tightly linked to memory performance. 


\section{Author notes}

The present study was supported by the National Natural Science Foundation of China (31671127),

Support by Capacity Building for Sci-Tech Innovation - Fundamental Scientific Research Funds (No.

025185305000/200). RT is supported by a British Academy Postdoctoral Fellowship (grant SUAI/028

RG94188). We thank Deborah Talmi and Zara Bergström for insightful discussion. The authors declare

no conflict of interest. 


\section{Open Practices Statement}

The studies reported in this manuscript were not preregistered. Upon publication, materials for the

experiments reported here are available on OSF (https://osf.io/7kpq6/). Data will be available upon request via the MRC Cognition and Brain Sciences Unit data repository. 


\section{References}

Addante, R. J., Ranganath, C., \& Yonelinas, A. P. (2012). Examining ERP correlates of recognition memory: Evidence of

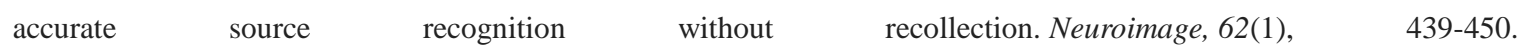
https://doi.org/10.1016/j.neuroimage.2012.04.031

Ahmad, F. N., \& Hockley, W. E. (2014). The role of familiarity in associative recognition of unitized compound word pairs. Quarterly Journal of Experimental Psychology, 67(12), 2301-2324. https://doi.org/10.1080/17470218.2014.923007

Bader, R. , \& Mecklinger, A. . (2017). Separating event-related potential effects for conceptual fluency and episodic familiarity. Journal of Cognitive Neuroscience, 29(8), 1402-1414. https://doi.org/10.1162/jocn_a_01131

Bader, R., Mecklinger, A., Hoppstädter, M., \& Meyer, P. (2010). Recognition memory for one-trial-unitized word pairs: Evidence from event-related potentials. Neuroimage, 50(2), 772-781. https://doi.org/10.1016/j.neuroimage.2009.12.100

Bastin, C., Besson, G., Simon, J., Delhaye, E., Geurten, M., Willems, S., \& Salmon, E. (2019). An Integrative Memory model of recollection and familiarity to understand memory deficits. Behavioral and Brain Sciences, 42. https://doi.org/10.1017/S0140525X19000621

Benjamini, Y., \& Hochberg, Y. (1995). Controlling the false discovery rate: a practical and powerful approach to multiple testing. Journal of the Royal statistical society: series B (Methodological), 57(1), 289-300. https://doi.org/10.1111/j.25176161.1995.tb02031.x

Bradley, M. M., Greenwald, M. K., Petry, M. C., \& Lang, P. J. (1992). Remembering pictures: pleasure and arousal in memory. Journal of experimental psychology: Learning, Memory, and Cognition, 18(2), 379. https://doi.org/10.1037/0278-7393.18.2.379

Cahill, L., \& McGaugh, J. L. (1995). A novel demonstration of enhanced memory associated with emotional arousal. Consciousness and cognition, 4(4), 410-421. https://doi.org/10.1006/ccog.1995.1048

Craik, F. I. (2002). Levels of processing: Past, present... and future?. Memory, 10(5-6), $305-318$. https://doi.org/10.1080/09658210244000135

Craik, F. I., \& Lockhart, R. S. (1972). Levels of processing: A framework for memory research. Journal of verbal learning and verbal behavior, 11(6), 671-684. https://doi.org/10.1016/S0022-5371(72)80001-X

Curran, T. (2000). Brain potentials of recollection and familiarity. Memory \& Cognition, 28(6), 923-938. 
https://doi.org/10.3758/BF03209340

Diana, R. A., Yonelinas, A. P., \& Ranganath, C. (2007). Imaging recollection and familiarity in the medial temporal lobe: a three-component model. Trends in Cognitive Sciences, 12(4), 379-386. https://doi.org/10.1016/j.tics.2007.08.001

Donaldson, D. I., \& Rugg, M. D. (1998). Recognition memory for new associations: electrophysiological evidence for the role of recollection. Neuropsychologia, 36(5), 377-395. https://doi.org/10.1016/S0028-3932(97)00143-7

Easterbrook, J. A. (1959). The effect of emotion on cue utilization and the organization of behavior. Psychological Review, 66(3), 183-201. https://doi.org/10.1037/h0047707

Engen, H. G., Smallwood, J., \& Singer, T. (2017). Differential impact of emotional task relevance on three indices of prioritised processing for fearful and angry facial expressions. Cognition \& Emotion, 31(1), 175-184. https://doi.org/10.1080/02699931.2015.1081873

Friedman, D. , Cycowicz, Y. M. , \& Bersick, M. . (2005). The late negative episodic memory effect: the effect of recapitulating study details at test. Brain Res Cogn Brain Res, 23(2-3), 185-198. https://doi.org/10.1016/j.cogbrainres.2004.10.005

Greve, A. , Rossum, M. , \& Donaldson, D. I. . (2007). Investigating the functional interaction between semantic and episodic memory: convergent behavioral and electrophysiological evidence for the role of familiarity. Neuroimage, 34(2), 801814. https://doi.org/10.1016/j.neuroimage.2006.07.043

Guillaume, F., \& Etienne, Y. (2015). Target- context unitization effect on the familiarity- related FN400: A face recognition exclusion task. International Journal of Psychophysiology, 95(3), 345-354. https://doi.org/10.1016/j.ijpsycho.2015.01.004

Guillet, R., \& Arndt, J. (2009). Taboo words: the effect of emotion on memory for peripheral information. Memory \& Cognition, 37(6), 866-879. https://doi.org/10.3758/MC.37.6.866

Han, M., Mao, X., Kartvelishvili, N., Li, W., \& Guo, C. (2018). Unitization mitigates interference by intrinsic negative emotion in familiarity and recollection of associative memory: Electrophysiological evidence. Cognitive, Affective, \& Behavioral Neuroscience, 8(6), 1259-1268. https://doi.org/10.3758/s13415-018-0636-y

Hou, M. , Safron, A. , Paller, K. A. , \& Guo, C. (2013). Neural correlates of familiarity and conceptual fluency in a recognition test with ancient pictographic characters. Brain Research, 1518, 48-60. https://doi.org/10.1016/j.brainres.2013.04.041

Huang, Y. M., Baddeley, A., \& Young, A. W. (2008). Attentional capture by emotional stimuli is modulated by semantic 
processing. Journal of Experimental Psychology: Human Perception and Performance, 34(2), 328-339. https://doi.org/10.1037/0096-1523.34.2.328

Humphreys, G. F. , Ralph, M. , \& Simons, J. S. . (2021). A unifying account of angular gyrus contributions to episodic and semantic cognition. Trends in Neurosciences, 44(6). https://doi.org/10.1016/j.tins.2021.01.006

Jäger, T., Mecklinger, A., \& Kipp, K. H. (2006). Intra-and inter-item associations doubly dissociate the electrophysiological correlates of familiarity and recollection. Neuron, 52(3), 535-545. https://doi.org/10.1016/j.neuron.2006.09.013

Johansson, M. , \& Mecklinger, A. . (2003). The late posterior negativity in erp studies of episodic memory: action monitoring and retrieval of attribute conjunctions. Biological Psychology, 64(1-2), 91-117. https://doi.org/10.1016/S03010511(03)00104-2

Kamp, S. M., Bader, R., \& Mecklinger, A. (2016). The effect of unitizing word pairs on recollection versus familiarity- based retrieval-Further evidence from ERPs. Advances in Cognitive Psychology, 12(4), 168-177. https://doi.org/10.5709/acp0196-2

Kensinger, E. A. (2009). Remembering the details: effects of emotion. Emotion Review, 1(2), 99-113. https://doi.org/10.1177/1754073908100432

Kensinger, E. A., \& Corkin, S. (2004). Two routes to emotional memory: Distinct neural processes for valence and arousal. Proceedings of the National Academy of Sciences, 101(9), 3310-3315. https://doi.org/10.1073/pnas.0306408101

Kriukova, O., Bridger, E., \& Mecklinger, A. (2013). Semantic relations differentially impact associative recognition memory: Electrophysiological evidence. Brain and Cognition, 83(1), 93-103. https://doi.org/10.1016/j.bandc.2013.07.006

LaBar, K. S., \& Phelps, E. A. (1998). Arousal-mediated memory consolidation: Role of the medial temporal lobe in humans. Psychological Science, 9(6), 490-493. https://doi.org/10.1111/1467-9280.00090

Lang, P. J., Bradley, M. M., N, B., \& Cuthbert. (2008). International Affective Picture System (IAPS): affective ratings of pictures and instruction manual (Rep. No. A-8).

Li, B., Han, M., Guo, C., \& Tibon, R. (2019). Unitization modulates recognition of within- domain and cross- domain associations: $\quad$ Evidence from event- related potentials. Psychophysiology, 56(11), e13446. https://doi.org/10.1111/psyp.13446

Li, B., Mao, X., Wang, Y., \& Guo, C. (2017). Electrophysiological correlates of familiarity and recollection in associative 
recognition: contributions of perceptual and conceptual processing to unitization. Frontiers in Human Neuroscience, 11, 125. https://doi.org/10.3389/fnhum.2017.00125

Liu, Z. , \& Guo, C. . (2019). Unitization improves item recognition through less overall neural processing. NeuroReport, 30(13), 882-886. https://doi.org/10.1097/WNR.0000000000001296

Liu, Z., Wu, J., Wang, Y., \& Guo, C. (2020). Unitization does not impede overall item recognition performance: Behavioral and event-related potential study. Neurobiology of Learning and Memory, 167, 107130. https://doi.org/10.1016/j.nlm.2019.107130

Lu, B., Liu, Z., Wang, Y., \& Guo, C. (2020). The different effects of concept definition and interactive imagery encoding on associative recognition for word and picture stimuli. International Journal of Psychophysiology, 158, 178-189. https://doi.org/10.1016/j.ijpsycho.2020.09.012

MacKay, D. G., Shafto, M., Taylor, J. K., Marian, D. E., Abrams, L., \& Dyer, J. R. (2004). Relations between emotion, memory, and attention: Evidence from taboo Stroop, lexical decision, and immediate memory tasks. Memory \& Cognition, 32(3), 474-488. https://doi.org/10.3758/BF03195840

Madan, C. R., Caplan, J. B., Lau, C. S. M., \& Fujiwara, E. (2012). Emotional arousal does not enhance association-memory. Journal of Memory \& Language, 66(4), 695-716. https://doi.org/10.1016/j.jml.2012.04.001

Mao, X., You, Y., Li, W., \& Guo, C. (2015). Emotion impairs extrinsic source memory--An ERP study. Biological Psychology, 110, 182-189. https://doi.org/10.1016/j.biopsycho.2015.07.005

Maratos, E. J., \& Rugg, M. D. (2001). Electrophysiological correlates of the retrieval of emotional and non-emotional context. Journal of Cognitive Neuroscience, 13(7), 877-91. https://doi.org/10.1162/089892901753165809

Mather, M. (2007). Emotional arousal and memory binding: An object-based framework. Perspectives on Psychological Science, 2(1), 33-52. https://doi.org/10.1111/j.1745-6916.2007.00028.x

Mather, M., \& Knight, M. (2008). The emotional harbinger effect: poor context memory for cues that previously predicted something arousing. Emotion, 8(6), 850-860. https://doi.org/10.1037/a0014087

Mather, M., \& Nesmith, K. (2008). Arousal-enhanced location memory for pictures. Journal of Memory \& Language, 58(2), 449-464. https://doi.org/10.1016/j.jml.2007.01.004

Mather, M., \& Sutherland, M. R. (2011). Arousal-biased competition in perception and memory. Perspectives on Psychological 
Science, 6(2), 114-133. https://doi.org/10.1177/1745691611400234

Mayes, A., Montaldi, D., \& Migo, E. (2007). Associative memory and the medial temporal lobes. Trends in cognitive sciences, 11(3), 126-135. https://doi.org/10.1016/j.tics.2006.12.003

Mecklinger, A. (2000). Interfacing mind and brain: A neurocognitive model of recognition memory. Psychophysiology, 37(5), 565-582. https://doi.org/10.1017/S0048577200992230

Mecklinger, A., \& Jäger, T. (2009). Episodic memory storage and retrieval: Insights from electrophysiological measures. Neuroimaging and psychological theories of human memory, 357-382. https://doi.org/10.1093/acprof:oso/9780199217298.003.0020

Mecklinger, A. , Rosburg, T. , \& Johansson, M. . (2016). Reconstructing the past: the late posterior negativity (lpn) in episodic memory studies. Neuroscience \& Biobehavioral Reviews, 68, 621-638. https://doi.org/10.1016/j.neubiorev.2016.06.024

Minor, G., \& Herzmann, G. (2019). Effects of negative emotion on neural correlates of item and source memory during encoding and retrieval. Brain Research, 1718, 32-45. https://doi.org/10.1016/j.brainres.2019.05.001

Molinaro, N., Vespignani, F. , Zamparelli, R. , \& Job, R. (2011). Why brother and sister are not just siblings: repair processes in agreement computation. Journal of Memory \& Language, 64(3), 211-232. https://doi.org/10.1016/j.jml.2010.12.002

Mollison, M. V. , \& Curran, T. . (2012). Familiarity in source memory. Neuropsychologia, 50(11), 2546-2565. https://doi.org/10.1016/j.neuropsychologia.2012.06.027

Murray, B. D., \& Kensinger, E. A. (2012). The effects of emotion and encoding strategy on associative memory. Memory \& Cognition, 40(7), 1056-1069. https://doi.org/10.3758/s13421-012-0215-3

Nashiro, K., \& Mather, M. (2011). Effects of emotional arousal on memory binding in normal aging and Alzheimer's disease. American Journal of Psychology, 124(3), 301-312. https://doi.org/10.5406/amerjpsyc.124.3.0301

Newsome, R.N., Dulas, M.R., \& Duarte, A. (2012). The effects of aging on emotion-induced modulations of source retrieval ERPs: $\quad$ evidence for valence biases. $\quad$ Neuropsychologia, $\quad 50, \quad 3370-3384$. https://doi.org/10.1016/j.neuropsychologia.2012.09.024

Opitz, B. . (2010). Neural binding mechanisms in learning and memory. Neuroscience \& Biobehavioral Reviews, 34(7), 10361046. https://doi.org/10.1016/j.neubiorev.2009.11.001

Paller, K. A., Ranganath, C., Gonsalves, B., LaBar, K. S., Parrish, T. B., Gitelman, D. R., ... \& Reber, P. J. (2003). Neural 
correlates of person recognition. Learning \& Memory, 10(4), 253-260. https://doi.org/10.1101/lm.57403

Paller, K. A., Voss, J. L., \& Boehm, S. G. (2007). Validating neural correlates of familiarity. Trends in cognitive sciences, 11(6), 243-250. https://doi.org/10.1016/j.tics.2007.04.002

Park, J. L. , \& Donaldson, D. I. . (2019). Detecting the neural correlates of episodic memory with mobile eeg: recollecting objects in the real world. NeuroImage, 193, 1-9. https://doi.org/10.1016/j.neuroimage.2019.03.013

Pessoa, L. (2009). How do emotion and motivation direct executive control? Trends in Cognitive Sciences, 13(4), 160-166. https://doi.org/10.1016/j.tics.2009.01.006

Pierce, B. H., \& Kensinger, E. A. (2011). Effects of emotion on associative recognition: valence and retention interval matter. Emotion, 11(1), 139-144. https://doi.org/10.1037/a0021287

Pilgrim, L. K., Murray, J. G., \& Donaldson, D. I. (2012). Characterizing episodic memory retrieval: Electrophysiological evidence for diminished familiarity following unitization. Journal of Cognitive Neuroscience, 24(8), 1671-1681. https://doi.org/10.1162/jocn_a_00186

Poldrack, R. A. (2006). Can cognitive processes be inferred from neuroimaging data?. Trends in cognitive sciences, 10(2), 5963. https://doi.org/10.1016/j.tics.2005.12.004

Rhodes, S. M. , \& Donaldson, D. I. . (2007). Electrophysiological evidence for the influence of unitization on the processes engaged during episodic retrieval: enhancing familiarity based remembering. Neuropsychologia, 45(2), 412-424.

Rhodes, S. M., \& Donaldson, D. I. (2008). Electrophysiological evidence for the effect of interactive imagery on episodic memory: encouraging familiarity for non-unitized stimuli during associative recognition. Neuroimage, 39(2), 873-884. https://doi.org/10.1016/j.neuroimage.2007.08.041

Riberto, M., Pobric, G., \& Talmi, D. (2019). The emotional facet of subjective and neural indices of similarity. Brain topography, 1-9. https://doi.org/10.1007/s10548-019-00743-7

Rimmele, U., Davachi, L., Petrov, R., Dougal, S., \& Phelps, E. A. (2011). Emotion enhances the subjective feeling of remembering, despite lower accuracy for contextual details. Emotion, 11(3), 553-562. https://doi.org/10.1037/a0024246

Rugg, M. D., \& Curran, T. (2007). Event-related potentials and recognition memory. Electroencephalography \& Clinical Neurophysiology, 72(5), 251-257. https://doi.org/10.1016/0013-4694(89)90045-X 
Schaefer, A., Pottage, C. L., \& Rickart, A. J. (2011). Electrophysiological correlates of remembering emotional pictures. NeuroImage, 54(1), 714-724. https://doi.org/10.1016/j.neuroimage.2010.07.030

Schmidt, K., Patnaik, P., \& Kensinger, E. A. (2011). Emotion's influence on memory for spatial and temporal context. Cognition \& Emotion, 25(2), 229-243. https://doi.org/10.1080/02699931.2010.483123

Smith, A., Dolan, R., \& Rugg, M. (2004). Event-related potential correlates of the retrieval of emotional and nonemotional context. Journal of Cognitive Neuroscience, 16(5), 760-775.

Snodgrass, J. G., \& Corwin, J. (1988). Pragmatics of measuring recognition memory: applications to dementia and amnesia. Journal of experimental psychology: General, 117(1), 34. https://doi.org/10.1037//0096-3445.117.1.34

Sommer, K. , Vita, S. , \& Pascalis, V. D. . (2018). The late posterior negativity in episodic memory: a correlate of stimulus retrieval? Biological Psychology, 133, 44-53. https://doi.org/10.1016/j.biopsycho.2018.01.016

Speer, N. K., \& Curran, T. (2007). ERP correlates of familiarity and recollection processes in visual associative recognition. Brain Research, 1174(1), 97-109. https://doi.org/10.1016/j.brainres.2007.08.024

Steinmetz, K. R., Knight, A. G., \& Kensinger, E. A. (2015). Neutral details associated with emotional events are encoded: evidence from a cued recall paradigm. Cognition \& Emotion, 30(7), 1352-1360. https://doi.org/10.1080/02699931.2015.1059317

Stein, T., Zwickel, J., Ritter, J., Kitzmantel, M., \& Schneider, W. X. (2009). The effect of fearful faces on the attentional blink is task dependent. Psychonomic Bulletin \& Review, 16(1), 104-109. https://doi.org/10.3758/PBR.16.1.104

Talmi, D., \& Moscovitch, M. (2004). Can semantic relatedness explain the enhancement of memory for emotional words?. Memory \& Cognition, 32(5), 742-751. https://doi.org/10.3758/BF03195864

Talmi, D., Schimmack, U., Paterson, T., \& Moscovitch, M. (2007). The role of attention and relatedness in emotionally enhanced memory. Emotion, 7(1), 89. https://doi.org/10.1037/1528-3542.7.1.89

Tibon, R., Ben-Zvi, S., \& Levy, D. A. (2014). Associative recognition processes are modulated by modality relations. Journal of Cognitive Neuroscience, 26(8), 1785-1796. https://doi.org/10.1162/jocn_a_00586

Tibon, R., Greve, A., \& Henson, R. (2018). The missing link? Testing a schema account of unitization. Memory \& cognition, 46(7), 1023-1040. https://doi.org/10.3758/s13421-018-0819-3

Tibon, R., Gronau, N., Scheuplein, A. L., Mecklinger, A., \& Levy, D. A. (2014). Associative recognition processes are 
bioRxiv preprint doi: https://doi.org/10.1101/2020.02.28.969659; this version posted October 4,2021 . The copyright holder for this preprint (which was not certified by peer review) is the author/funder, who has granted bioRxiv a license to display the preprint in perpetuity. It is made available under aCC-BY 4.0 International license.

EMOTION AND RELATEDNESS MODULATE MEMORY

modulated by the semantic unitizability of memoranda. Brain \& Cognition, 92, 19-31. https://doi.org/10.1016/j.bandc.2014.09.009

Ventura-Bort, C., Löw, A., Wendt, J. et al. (2016). Binding neutral information to emotional contexts: Brain dynamics of longterm recognition memory. Cogn Affect Behav Neurosci 16, 234-247 . https://doi.org/10.3758/s13415-015-0385-0

Vilberg, K. L. , \& MD Rugg. (2008). Memory retrieval and the parietal cortex: a review of evidence from a dual-process perspective. Neuropsychologia, 46(7), 1787-1799. https://doi.org/10.1016/j.neuropsychologia.2008.01.004

Vilberg, K. L. , \& MD Rugg. (2012). The neural correlates of recollection: transient versus sustained fmri effects. Journal of Neuroscience, 32(45), 15679. https://doi.org/10.1523/JNEUROSCI.3065-12.2012

Vilberg, K. L. , \& MD Rugg. (2013). Temporal dissociations within the core recollection network. Cognitive Neuroscience, 5(2), 77-84. https://doi.org/10.1080/17588928.2013.860088

Wei, P., Wang, D., \& Ji, L. (2015). Reward expectation regulates brain responses to task-relevant and task-irrelevant emotional words: ERP evidence. Social Cognitive \& Affective Neuroscience, 11(2), 191-203. https://doi.org/10.1093/scan/nsv097

Weymar, M., Löw, A., \& Hamm, A. O. (2011). Emotional memories are resilient to time: Evidence from the parietal ERP old/new effect. Human Brain Mapping, 32(4), 632-640. https://doi.org/10.1002/hbm.21051

Weymar, M., Löw, A., Schwabe, L., \& Hamm, A. O. (2010). Brain dynamics associated with recollective experiences of emotional events. Neuroreport, 21(12), 827-831. https://doi.org/10.1097/WNR.0b013e32833d180a

Wilding, E. L., \& Ranganath, C. (2011). Electrophysiological correlates of episodic memory processes. In S. J. Luck \& E. Kappenman (Eds.), The Oxford Handbook of ERP Components (pp. 373-396). Oxford: Oxford University Press. https://doi.org/10.1093/oxfordhb/9780195374148.001.0001

Wolk, D. A., Schacter, D. L., Lygizos, M., Sen, N. M., Holcomb, P. J., Daffner, K. R., \& Budson, A. E. (2006). ERP correlates of recognition memory: effects of retention interval and false alarms. Brain Research, 1096(1), 148-162. https://doi.org/10.1016/j.brainres.2006.04.050

Yonelinas, A. P. (2002). The nature of recollection and familiarity: a review of 30 years of research. Journal of Memory \& Language, 46(3), 441-517. https://doi.org/10.1006/jmla.2002.2864

Yonelinas, A. P., Aly, M., Wang, W. C., \& Koen, J. D. (2010). Recollection and familiarity: Examining controversial assumptions and new directions. Hippocampus, 20(11), 1178-1194. https://doi.org/10.1002/hipo.20864 
Zhao, M. F. , Zimmer, H. D. , Fu, X. , \& Zheng, Z. . (2020). Unitization of internal and external features contributes to associative recognition for faces: evidence from modulations of the fn400. Brain Research, 1748, 147077. https://doi.org/10.1016/j.brainres.2020.147077

Zheng, Z. , Li, J. , Xiao, F. , Broster, L. S. , \& Jiang, Y. . (2015). Electrophysiological evidence for the effects of unitization on associative recognition memory in older adults. Neurobiology of Learning \& Memory, 121, 59-71. https://doi.org/10.1016/j.nlm.2015.03.006

Zheng, Z., Li, J., Xiao, F., Broster, L. S., Jiang, Y., \& Xi, M. (2015). The effects of unitization on the contribution of familiarity and recollection processes to associative recognition memory: evidence from event-related potentials. International Journal of Psychophysiology, 95(3), 355-362. https://doi.org/10.1016/j.ijpsycho.2015.01.003

Zheng, Z., Li, J., Xiao, F., Ren, W., \& He, R. (2016). Unitization improves source memory in older adults: an event-related potential study. Neuropsychologia, 89, 232-244. https://doi.org/10.1016/j.neuropsychologia.2016.06.025 